\title{
Data Integration with Uncertainty
}

\author{
Xin Dong \\ University of Washington \\ Seattle, WA 98195 \\ lunadong@cs.washington.edu
}

\author{
Alon Y. Halevy \\ Google Inc. \\ Mountain View, CA 94043 \\ halevy@google.com
}

\author{
Cong $\mathrm{Yu}$ \\ University of Michigan \\ Ann Arbor, MI 48109 \\ congy@eecs.umich.edu
}

July 28, 2007

\begin{abstract}
This paper reports our first set of results on managing uncertainty in data integration. We posit that data-integration systems need to handle uncertainty at three levels, and do so in a principled fashion. First, the semantic mappings between the data sources and the mediated schema may be approximate because there may be too many of them to be created and maintained or because in some domains (e.g., bioinformatics) it is not clear what the mappings should be. Second, queries to the system may be posed with keywords rather than in a structured form. Third, the data from the sources may be extracted using information extraction techniques and so may yield erroneous data.

As a first step to building such a system, we introduce the concept of probabilistic schema mappings and analyze their formal foundations. We show that there are two possible semantics for such mappings: by-table semantics assumes that there exists a correct mapping but we don't know what it is; by-tuple semantics assumes that the correct mapping may depend on the particular tuple in the source data. We present the query complexity and algorithms for answering queries in the presence of approximate schema mappings, and we describe an algorithm for efficiently computing the top-k answers to queries in such a setting.
\end{abstract}

\section{Introduction}

Data integration and exchange systems offer a uniform interface to a multitude of data sources and the ability to share data across multiple systems. These systems have recently enjoyed significant research and commercial success $[15,13]$ Current data integration systems are essentially a natural extension of traditional database systems in that queries are specified in a structured form and data are modeled in one of the traditional data models (relational, XML). In addition, the data integration system has exact knowledge of how the data in the sources map to the schema used by the data integration system.

We argue that as the scope of data integration applications broadens, such systems need to be able to model uncertainty at their core. Uncertainty can arise for multiple reasons in data integration. First, the semantic mappings between the data sources and the mediated schema may be approximate. For example, in an application like Google Base or when mapping millions of sources on the deep web [20], we cannot imagine specifying exact mappings. In some domains (e.g., bioinformatics), we do not necessarily know what the exact mapping is. Second, if the intended users of the application are not necessarily familiar with schemata, or if the domain of the system is too broad to offer form-based query interfaces (such as web forms), we need to support keyword queries. Hence, a second source of uncertainty is the transformation between keyword queries and 
a set of candidate structured queries. Finally, data is often extracted from unstructured sources using information extraction techniques. Since these techniques are approximate, the data obtained from the sources may be uncertain.

Dataspace Support Platforms [14] envision data integration systems where sources are added with no effort and the system is constantly evolving in a pay-as-you-go fashion to improve the quality of semantic mappings and query answering. Enabling data integration with uncertainty is a key technology to supporting dataspaces.

This paper takes a first step towards the goal of data integration with uncertainty. We first describe how the architecture of such a system differs from a traditional one (Section 2). At the core, the system models tuples and semantic mappings with probabilities associated with them. Query answering ranks answers and typically tries to obtain the top-k results to a query. These changes lead to a requirement for a new kind of adaptivity in query processing.

We then focus on one core component of data integration with uncertainty, namely probabilistic schema mappings (Section 3). Semantic mappings are the component of a data integration system that specify the relationship between the contents of the different sources. The mappings enable the data integration to reformulate a query posed over the mediated schema into queries over the sources $[17,12]$. We introduce probabilistic schema mappings, and describe how to answer queries in their presence.

We define probabilistic schema mapping as a set of possible (ordinary) mappings between a source schema and a target schema, where each possible mapping has an associated probability. To focus on the key issues, we begin by considering a simple class of mappings, where each mapping describes a set of correspondences between the attributes of a source table and the attributes of a target table. We argue that there are two possible interpretations of probabilistic schema mappings. In the first, which we formalize as by-table semantics, we assume there exists a single correct mapping between the source and the target, but we don't know which one it is. In the second, called by-tuple semantics, the correct mapping may depend on the particular tuple in the source to which it is applied. In both cases, the semantics of query answers are a generalization of certain answers [1] for data integration systems.

We describe algorithms for answering queries in the presence of probabilistic schema mappings and then analyze the computational complexity of answering queries (Section 4). We show that the data complexity of answering queries in the presence of probabilistic mappings is PTIME for by-table semantics and \#P-complete for by-tuple semantics. We identify a large subclass of realworld queries for which we can still obtain all the by-tuple answers in PTIME. We then describe algorithms for finding the top-k answers to a query (Section 5).

The size of a probabilistic mapping may be quite large, since it essentially enumerates a probability distribution by listing every combination of events in the probability space. In practice, we can often encode the same probability distribution much more concisely. Our next contribution (Section 6) is to identify two concise representations of probabilistic mappings for which query answering can be done in PTIME in the size of the mapping. We also examine the possibility of representing a probabilistic mapping as a Bayes Net, but show that query answering may still be exponential in the size of a Bayes Net representation of a mapping.

We then consider using probabilistic mappings in the scenario of data exchange (Section 7), and show that we can create a probabilistic database representing a core universal solution in linear time. As in the case of non-probabilistic mappings, the core universal solution can be used to find all the answers to a given query.

Finally, we consider several more powerful mapping languages, such as complex mappings, where 


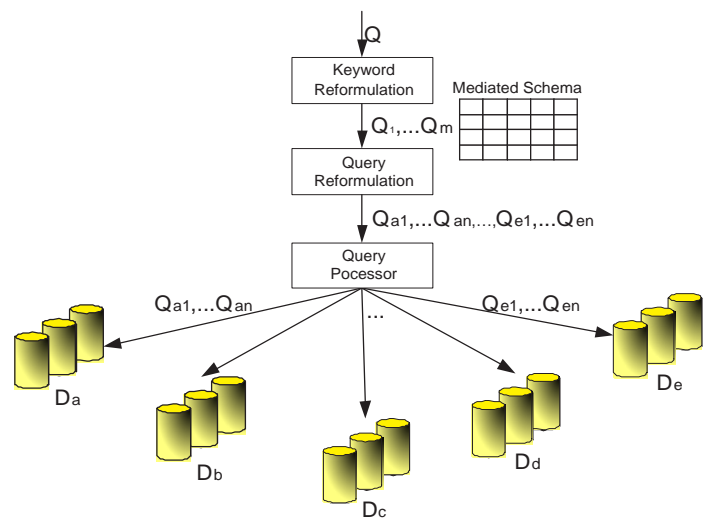

Figure 1: Architecture of a data-integration system that handles uncertainty.

the correspondences are between sets of attributes, and conditional mappings, where the mapping is conditioned on a property of the tuple to which it is applied (Section 8).

\section{Overview of the System}

This section describes the requirements from a data integration system that supports uncertainty and the overall architecture of the system. We frame our specific contributions in context of this architecture.

\subsection{Uncertainty in Data Integration}

A data integration system needs to handle uncertainty at three levels.

Uncertain schema mappings: Data integration systems rely on schema mappings for specifying the semantic relationships between the data in the sources and the terms used in the mediated schema. However, schema mappings can be inaccurate. In many applications it is impossible to create and maintain precise mappings between data sources. This can be because the users are not skilled enough to provide precise mappings, such as in personal information management [7], because people do not understand the domain well and thus do not even know what correct mappings are, such as in bioinformatics, or because the scale of the data prevents generating and maintaining precise mappings, such as in integrating data of the web scale [20]. Hence, in practice, schema mappings are often generated by semi-automatic tools, and not necessarily verified by domain experts.

Uncertain data: By nature, data integration systems need to handle uncertain data. One reason for uncertainty is that data is often extracted from unstructured or semi-structured sources by automatic methods (e.g., HTML pages, emails, blogs). A second reason is that data may come from sources that are unreliable or not up to date.

Uncertain queries: In some data integration applications, especially on the web, queries will be posed as keywords rather than as structured queries against a well defined schema. The system needs to translate these queries into some structured form so they can be reformulated with respect to the data sources. At this step, the system may generate multiple candidate structured queries and have some uncertainty about which is the real intent of the user. 


\subsection{System Architecture}

Given the above requirements, we describe the architecture of a data integration system that manages uncertainty at its core. We describe the system by contrasting it to a traditional data integration system.

The first and most fundamental characteristic of this system is that it is based on a probabilistic data model. This means two things. First, as we process data in the system we attach probabilities to each tuple. Second, and the focus of this paper, is that schema mappings are also associated with probabilities, modeling our uncertainty about which ones are correct. We note that the probabilities associated with tuples, mappings, and answers are mostly internal to the system, and are not meant to be exposed to users. Typically, we will use these probabilities to rank answers.

Second, whereas traditional data integration systems begin by reformulating a query onto the schemas of the data sources, a data integration system with uncertainty needs to first reformulate a keyword query into a set of candidate structured queries. We refer to this step as keyword reformulation. Note that keyword reformulation is different from techniques for keyword search on structured data (e.g., $[16,2])$ in that (a) it does not assume access to all the data in the sources or that the sources support keyword search, and (b) it tries to distinguish different structural elements in the query in order to pose more precise queries to the sources (e.g., realizing that in the keyword query "chicago weather", "weather" is an attribute label and "chicago" is an instance name). That being said, keyword reformulation should benefit from techniques that support answering keyword search on structured data.

Third, the query answering model is different. Instead of necessarily finding all answers to a given query, our goal is typically to find the top-k answers, and rank these answers most effectively.

The final difference from traditional data integration systems is that our query processing will need to be more adaptive than usual. Instead of generating a query answering plan and executing it, the steps we take in query processing will depend on results of previous steps. We note that adaptive query processing has been discussed quite a bit in data integration [18], where the need for adaptivity arises from the fact that data sources did not answer as quickly as expected or that we did not have accurate statistics about their contents to properly order our operations. In our work, however, the goal for adaptivity is to get the answers with high probabilities faster.

The architecture of the system is shown in Figure 1. The system contains a number of data sources and a mediated schema. When the user poses a query $Q$, which can be either a structured query on the mediated schema, or a keyword query, the system returns a set of answer tuples, each with a probability. If $Q$ is a keyword query, the system first performs keyword reformulation to translate it into a set of candidate structured queries on the mediated schema. Otherwise, the candidate query is $Q$ itself.

Consider how the system answers the candidate queries, and assume the queries will not involve joins over multiple sources. For each candidate structured query $Q_{0}$ and a data source $S$, the system reformulates $Q_{0}$ according to the schema mapping (which can be uncertain) between $S$ 's schema and the mediated schema, sends the reformulated query (or queries) to $S$, retrieving the answers. If the user asks for all the answers to the query, then the reformulated query is typically a query with grouping and aggregation. If $S$ does not support grouping and aggregation, then grouping and aggregation can be processed in the integration system. If the user asks for top-k answers, then query processing is more complex. The system reformulates the query into a set of queries, and uses a middle layer to decide at runtime which queries are critical to computing the top- $k$ answers and sends the appropriate queries to $S$. Note that there can be multiple iterations of deciding the promising reformulated queries and retrieving answers. Furthermore, the system can even decide 


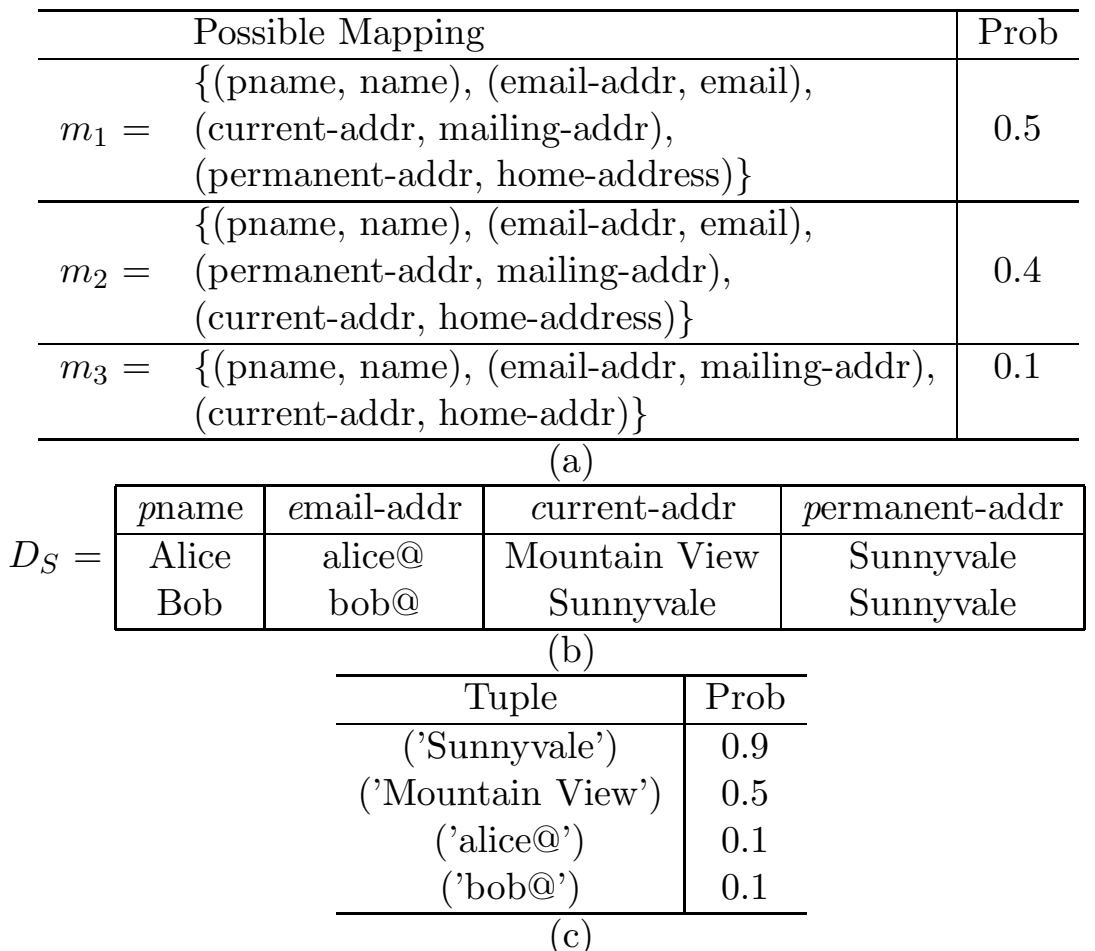

Figure 2: The running example: (a) a probabilistic schema mapping between $S$ and $T$; (b) a source instance $D_{S}$; (c) the answers of $Q$ over $D_{S}$ with respect to the probabilistic mapping.

which data sources are more relevant and prioritize the queries to those data sources. Finally, if the data in the sources are uncertain, then the sources will return answers with probabilities attached to them.

After receiving answers from different data sources, the system combines them to get one single set of answer tuples. For example, if the data sources are known to be independent of each other, and we obtain tuple $t$ from $n$ data sources with probabilities $p_{1}, \ldots, p_{n}$ respectively, then in the final answer set $t$ has probability $1-\Pi_{i=1}^{n}\left(1-p_{i}\right)$. If we know that some data sources are duplicates or extensions of others, a different combination function needs to be used.

\subsection{Handling Uncertainty in Mappings}

As a first step towards developing such a data integration system, we introduce in this paper probabilistic schema mappings, and show how to answer queries in their presence. Before the formal discussion, we illustrate the main ideas with an example.

Example 2.1. Consider a data source $S$, which describes a person by her email address, current address, and permanent address, and the mediated schema $T$, which describes a person by her name, email, mailing address, home address and office address:

$\mathrm{S}=$ (pname, email-addr, current-addr, permanent-addr)

$\mathrm{T}=$ (name, email, mailing-addr, home-addr, office-addr)

A semi-automatic schema-mapping tool may generate three possible mappings between $S$ and $T$, assigning each a probability. Whereas the three mappings all map pname to name, they map other attributes in the source and the target differently. Figure 2(a) describes the three mappings using sets of attribute correspondences. For example, mapping $m_{1}$ maps pname to name, email-addr to 
email, current-addr to mailing-addr, and permanent-addr to home-addr. Because of the uncertainty on which mapping is correct, we consider all of these mappings in query answering.

Suppose the system receives a query $Q$ composed on the mediated schema and asking for people's mailing addresses:

Q: SELECT mailing-addr FROM T

Using the possible mappings, we can reformulate $Q$ into different queries:

Q1: SELECT current-addr FROM $\mathrm{S}$

Q2: SELECT permanent-addr FROM $S$

Q3: SELECT email-addr FROM S

If the user requires all possible answers, the system generates a single aggregation query based on $Q_{1}, Q_{2}$ and $Q_{3}$ to compute the probability of each returned tuple, and sends the query to the data source. Suppose the data source contains a table $D_{S}$ as shown in Figure 2(b), the system will retrieve four answer tuples, each with a probability, as shown in Figure 2(c).

If the user requires only the top-1 answer (i.e., the answer tuple with the highest probability), the system decides at runtime which reformulated queries to execute. For example, after executing $Q_{1}$ and $Q_{2}$ at the source, the system can already conclude that ('Sunnyvale') is the top-1 answer and can skip query $Q_{3}$.

\subsection{Source of probabilities}

A critical issue in any system that manages uncertainty is whether we have a reliable source of probabilities. Whereas obtaining reliable probabilities for such a system is one of the most interesting areas for future research, there is quite a bit to build on. For keyword reformulation, it is possible to train and test reformulators on large numbers of queries such that each reformulation result is given a probability based on its performance statistics. In the case of schema matching, it is standard practice for schema matchers to also associate numbers with the candidates they propose. The issue here is that the numbers are meant only as a ranking mechanism rather than true probabilities. However, as schema matching techniques start looking a larger number of schemas, one can imagine ascribing probabilities (or approximations thereof) to their measures. Finally, information extraction techniques are also often based on statistical machine learning methods, thereby lending their predictions a probabilistic interpretation.

\section{Probabilistic Schema Mapping}

In this section we formally define the semantics of probabilistic schema mappings and the query answering problems we consider. Our discussion is in the context of the relational data model. A schema contains a finite set of relations. Each relation contains a finite set of attributes and is denoted by $R=\left\langle r_{1}, \ldots, r_{n}\right\rangle$. An instance $D_{R}$ of $R$ is a finite set of tuples, where each tuple associates a value with each attribute in the schema.

We consider select-project-join (SPJ) queries in SQL. Note that answering such queries is in PTIME in the size of the data.

\subsection{Schema Mappings}

We begin by reviewing non-probabilistic schema mappings. The goal of a schema mapping is to specify the semantic relationships between a source schema and a target schema. We refer to the 
source schema as $\bar{S}$, and a relation in $\bar{S}$ as $S=\left\langle s_{1}, \ldots, s_{m}\right\rangle$. Similarly, we refer to the target schema as $\bar{T}$, and a relation in $\bar{T}$ as $T=\left\langle t_{1}, \ldots, t_{n}\right\rangle$.

The common formalism for schema mappings, GLAV, is based on expressions of the form

$$
m: \forall \mathbf{x}(\phi(\mathbf{x}) \rightarrow \exists \mathbf{y} \psi(\mathbf{x}, \mathbf{y})) .
$$

In the expression, $\phi$ is the body of a conjunctive query over $\bar{S}$ and $\psi$ is the body of a conjunctive query over $\bar{T}$. A pair of instances $D_{S}$ and $D_{T}$ satisfies a GLAV mapping $m$ if for every assignment of $\mathbf{x}$ in $D_{S}$ that satisfies $\phi$ there exists an assignment of $\mathbf{y}$ in $D_{T}$ that satisfies $\psi$.

We consider a limited form of GLAV mappings where each side of the mapping involves only projection queries on a single table. These mappings have also been referred to as schema matching in the literature [22]. Specifically, we consider GLAV mappings where (1) $\phi$ (resp. $\psi$ ) is an atomic formula over $S$ (resp. T), (2) the GLAV mapping does not include constants, and (3) each variable occurs at most once on each side of the mapping. We consider this class of mappings because they already expose many of the novel issues involved in probabilistic mappings and because they are quite common in practice. We also note that many of the concepts we define apply to a broader class of mappings, which we will discuss in detail in Section 8.

Given these restrictions, we can define our mappings in terms of attribute correspondences. An attribute correspondence is of the form $c_{i j}=\left(s_{i}, t_{j}\right)$, where $s_{i}$ is a source attribute in the schema $S$ and $t_{j}$ is a target attribute in the schema $T$. Intuitively, $c_{i j}$ specifies that there is a relationship between $s_{i}$ and $t_{j}$. In practice, a correspondence also involves a function that transforms the value of $s_{i}$ to the value of $t_{j}$. For example, the correspondence (c-degree, temperature) can be specified as temperature $=\mathrm{c}$-degree $* 1.8+32$, describing a transformation from Celsius to Fahrenheit. These functions are irrelevant to our discussion, and therefore we omit them. Formally, we define relation mappings and schema mappings as follows.

Definition 3.1 (Schema Mapping). Let $\bar{S}$ and $\bar{T}$ be relational schemas. A relation mapping $M$ is a triple $(S, T, m)$, where $S$ is a relation in $\bar{S}, T$ is a relation in $\bar{T}$, and $m$ is a set of attribute correspondences between $S$ and $T$.

When each source and target attribute occurs in at most one correspondence in $m$, we call $M a$ one-to-one relation mapping.

A schema mapping $\bar{M}$ is a set of one-to-one relation mappings between relations in $\bar{S}$ and in $\bar{T}$, where every relation in either $\bar{S}$ or $\bar{T}$ appears at most once.

Example 3.2. Consider the mappings in Example 2.1. We can view $m_{1}$ as a GLAV mapping:

$$
\forall n, e, c, p(S(n, e, c, p) \rightarrow \exists o(T(n, e, c, p, o)))
$$

The database in Figure 2(b) (repeated in Figure 3(a)) and the database in Figure 3(b) satisfy $m_{1}$.

\subsection{Probabilistic Schema Mappings}

Intuitively, a probabilistic schema mapping describes a probability distribution of a set of possible schema mappings between a source schema and a target schema.

Definition 3.3 (Probabilistic Mapping). Let $\bar{S}$ and $\bar{T}$ be relational schemas. A probabilistic mapping (p-mapping), $p M$, is a triple $(S, T, \mathbf{m})$, where $S \in \bar{S}, T \in \bar{T}$, and $\mathbf{m}$ is a set $\left\{\left(m_{1}, \operatorname{Pr}\left(m_{1}\right)\right), \ldots\right.$, $\left.\left(m_{l}, \operatorname{Pr}\left(m_{l}\right)\right)\right\}$, such that 


\begin{tabular}{|c|c|c|c|}
\hline pname & email-addr & permanent-addr & current-addr \\
\hline Alice & alice@ & Mountain View & Sunnyvale \\
Bob & bob@ & Sunnyvale & Sunnyvale \\
\hline
\end{tabular}

(a)

\begin{tabular}{|c|c|c|c|c|}
\hline name & email & mailing-addr & home-addr & office-addr \\
\hline Alice & alice@ & Mountain View & Sunnyvale & office \\
Bob & bob@ & Sunnyvale & Sunnyvale & office \\
\hline
\end{tabular}

(b)

\begin{tabular}{|c|c|c|c|c|}
\hline name & email & mailing-addr & home-addr & office-addr \\
\hline Alice & alice@ & Sunnyvale & Mountain View & office \\
Bob & email & bob@ & Sunnyvale & office \\
\hline
\end{tabular}

(c)

\begin{tabular}{c|c}
\hline Tuple & Prob \\
\hline ('Sunnyvale') & 0.9 \\
('Mountain View') & 0.5 \\
('alice@') & 0.1 \\
('bob@') & 0.1 \\
\hline
\end{tabular}

(d)

\begin{tabular}{c|c}
\hline Tuple & Prob \\
\hline ('Sunnyvale') & 0.94 \\
('Mountain View') & 0.5 \\
('alice@') & 0.1 \\
('bob@') & 0.1 \\
\hline
\end{tabular}

Figure 3: Example 3.10: (a) a source instance $D_{S}$; (b) a target instance that is by-table consistent with $D_{S}$; (c) a target instance that is by-tuple consistent with $D_{S}$; (d) $Q^{\text {table }}\left(D_{S}\right)$; (e) $Q^{\text {tuple }}\left(D_{S}\right)$.

- for $i \in[1, l], m_{i}$ is a one-to-one mapping between $S$ and $T$, and for every $i, j \in[1, l], i \neq j \Rightarrow$ $m_{i} \neq m_{j}$.

- $\operatorname{Pr}\left(m_{i}\right) \in[0,1]$ and $\sum_{i=1}^{l} \operatorname{Pr}\left(m_{i}\right)=1$.

A schema p-mapping, $\overline{p M}$, is a set of p-mappings between relations in $\bar{S}$ and in $\bar{T}$, where every relation in either $\bar{S}$ or $\bar{T}$ appears in at most one p-mapping.

Note that we assume the possible mappings in a p-mapping are independent. We refer to a non-probabilistic mapping as an ordinary mapping. A schema p-mapping may contain both pmappings and ordinary mappings. Example 2.1 shows a p-mapping (see Figure 2(a)) that contains three possible mappings.

\subsection{Semantics of Probabilistic Mappings}

Intuitively, a probabilistic schema mapping models the uncertainty about which of the mappings in $p M$ is the correct one. When a schema matching system produces a set of candidate matches, there are two ways to interpret the uncertainty: (1) a single mapping in $p M$ is the correct one and it applies to all the data in $S$, or (2) multiple mappings are correct and each suitable for a subset of tuples in $S$, though it is not known which mapping is the right one for a specific tuple. Example 2.1 illustrates the first interpretation. For the same example, the second interpretation is equally valid: some people may choose to use their current address as mailing address while others use their permanent address as mailing address; thus, for different tuples we may apply different mappings, so the correct mapping depends on the particular tuple.

This paper analyzes query answering under both interpretations. We refer to the first interpretation as the by-table semantics and to the second one as the by-tuple semantics of probabilistic 
mappings. We are not trying to argue for one interpretation over the other. The needs of the application should dictate the appropriate semantics. Furthermore, our complexity results, which will show advantages to by-table semantics, should not be taken as an argument in the favor of by-table semantics.

We next define the semantics of p-mappings in detail and the definitions for schema p-mappings are the obvious extensions. The semantics of p-mappings is defined as a natural extension of that of ordinary mappings, which we review now. A mapping defines a relationship between instances of $S$ and instances of $T$ that are consistent with the mapping.

Definition 3.4 (Consistent Target Instance). Let $M=(S, T, m)$ be a relation mapping and $D_{S}$ be an instance of $S$.

An instance $D_{T}$ of $T$ is said to be consistent with $D_{S}$ and $M$, if for each tuple $t_{s} \in D_{S}$, there exists a tuple $t_{t} \in D_{T}$, such that for every attribute correspondence $\left(a_{s}, a_{t}\right) \in m$, the value of $a_{s}$ in $t_{s}$ is the same as the value of $a_{t}$ in $t_{t}$.

For a relation mapping $M$ and a source instance $D_{S}$, there can be an infinite number of target instances that are consistent with $D_{S}$ and $M$. We denote by $\operatorname{Tar}_{M}\left(D_{S}\right)$ the set of all such target instances. The set of answers to a query $Q$ is the intersection of the answers on all instances in $\operatorname{Tar}_{M}\left(D_{S}\right)$. The following definition is from [1].

Definition 3.5 (Certain Answer). Let $M=(S, T, m)$ be a relation mapping. Let $Q$ be a query over $T$ and let $D_{S}$ be an instance of $S$.

A tuple $t$ is said to be a certain answer of $Q$ with respect to $D_{S}$ and $M$, if for every instance $D_{T} \in \operatorname{Tar}_{M}\left(D_{S}\right), t \in Q\left(D_{T}\right)$.

By-table semantics: We now generalize these notions to the probabilistic setting, beginning with the by-table semantics. Intuitively, a p-mapping $p M$ describes a set of possible worlds, each with a possible mapping $m \in p M$. In by-table semantics, a source table can fall in one of the possible worlds; that is, the possible mapping associated with that possible world applies to the whole source table. Following this intuition, we define target instances that are consistent with the source instance.

Definition 3.6 (By-table Consistent Instance). Let $p M=(S, T, \mathbf{m})$ be a p-mapping and $D_{S}$ be an instance of $S$.

An instance $D_{T}$ of $T$ is said to be by-table consistent with $D_{S}$ and $p M$, if there exists a mapping $m \in \mathbf{m}$ such that $D_{S}$ and $D_{T}$ satisfy $m$.

Given a source instance $D_{S}$ and a possible mapping $m \in \mathbf{m}$, there can be an infinite number of target instances that are consistent with $D_{S}$ and $m$. We denote by $\operatorname{Tar}_{m}\left(D_{S}\right)$ the set of all such instances.

In the probabilistic context, we assign a probability to every answer. Intuitively, we consider the certain answers with respect to each possible mapping in isolation. The probability of an answer $t$ is the sum of the probabilities of the mappings for which $t$ is deemed to be a certain answer. We define by-table answers as follows:

Definition 3.7 (By-table Answer). Let $p M=(S, T, \mathbf{m})$ be a p-mapping. Let $Q$ be a query over $T$ and let $D_{S}$ be an instance of $S$.

Let $t$ be a tuple. Let $\bar{m}(t)$ be the subset of $\mathbf{m}$, such that for each $m \in \bar{m}(t)$ and for each $D_{T} \in \operatorname{Tar}_{m}\left(D_{S}\right), t \in Q\left(D_{T}\right)$. 
Let $p=\sum_{m \in \bar{m}(t)} \operatorname{Pr}(m)$. If $p>0$, then we say $(t, p)$ is a by-table answer of $Q$ with respect to $D_{S}$ and $p M$.

By-tuple semantics: If we follow the possible-world notions, in by-tuple semantics, different tuples in a source table can fall in different possible worlds; that is, different possible mappings associated with those possible worlds can apply to the different source tuples.

Formally, the key difference in the definition of by-tuple semantics from that of by-table semantics is that a consistent target instance is defined by a mapping sequence that assigns a (possibly different) mapping in $\mathbf{m}$ to each tuple in $D_{S}$. (Without losing generality, in order to compare between such sequences, we assign some order to the tuples in the instance).

Definition 3.8 (By-tuple Consistent Instance). Let $p M=(S, T, \mathbf{m})$ be a p-mapping and let $D_{S}$ be an instance of $S$ with d tuples.

An instance $D_{T}$ of $T$ is said to be by-tuple consistent with $D_{S}$ and $p M$, if there is a sequence $\left\langle m^{1}, \ldots, m^{d}\right\rangle$ such that for every $1 \leq i \leq d$,

- $m^{i} \in \mathbf{m}$, and

- for the $i^{\text {th }}$ tuple of $D_{S}, t_{i}$, there exists a target tuple $t_{i}^{\prime} \in D_{T}$ such that for each attribute correspondence $\left(a_{s}, a_{t}\right) \in m^{i}$, the value of $a_{s}$ in $t_{i}$ is the same as the value of $a_{t}$ in $t_{i}^{\prime}$.

Given a mapping sequence $s e q=\left\langle m^{1}, \ldots, m^{d}\right\rangle$, we denote by $\operatorname{Tar}_{s e q}\left(D_{S}\right)$ the set of all target instances that are consistent with $D_{S}$ and seq. Note that if $D_{T}$ is by-table consistent with $D_{S}$ and $m$, then $D_{T}$ is also by-tuple consistent with $D_{S}$ and a mapping sequence in which each mapping is $m$.

We can think of every sequence of mappings $s e q=\left\langle m^{1}, \ldots, m^{d}\right\rangle$ as a separate event whose probability is $\operatorname{Pr}(\mathrm{seq})=\prod_{i=1}^{d} \operatorname{Pr}\left(m^{i}\right)$. (In Section 8 we relax this independence assumption and introduce conditional mappings.) If there are $l$ mappings in $p M$, then there are $l^{d}$ sequences of length $d$, and their probabilities add up to 1 . We denote by $\mathbf{s e q}_{d}(p M)$ the set of mapping sequences of length $d$ generated from $p M$.

Definition 3.9 (By-tuple Answer). Let $p M=(S, T, \mathbf{m})$ be a p-mapping. Let $Q$ be a query over $T$ and $D_{S}$ be an instance of $S$ with d tuples.

Let $t$ be a tuple. Let $\overline{\operatorname{seq}}(t)$ be the subset of $\mathbf{s e q}_{d}(p M)$, such that for each seq $\in \overline{\operatorname{seq}}(t)$ and for each $D_{T} \in \operatorname{Tar}_{\text {seq }}\left(D_{S}\right), t \in Q\left(D_{T}\right)$.

Let $p=\sum_{s e q \in \overline{s e q}(t)} \operatorname{Pr}(s e q)$. If $p>0$, we call $(t, p)$ a by-tuple answer of $Q$ with respect to $D_{S}$ and $p M$.

The set of by-table answers for $Q$ with respect to $D_{S}$ is denoted by $Q^{\text {table }}\left(D_{S}\right)$ and the set of by-tuple answers for $Q$ with respect to $D_{S}$ is denoted by $Q^{\text {tuple }}\left(D_{S}\right)$.

Example 3.10. Consider the p-mapping $p M$, the source instance $D_{S}$, and the query $Q$ in the motivating example.

In by-table semantics, Figure 3(b) shows a target instance that is consistent with $D_{S}$ (repeated in Figure 3(a)) and possible mapping $m_{1}$. Figure 3(d) shows the by-table answers of $Q$ with respect to $D_{S}$ and $p M$. As an example, for tuple $t=\left(\right.$ 'Sunnyvale'), we have $\bar{m}(t)=\left\{m_{1}, m_{2}\right\}$, so the possible tuple ('Sunnyvale', 0.9) is an answer.

In by-tuple semantics, Figure 3(c) shows a target instance that is by-tuple consistent with $D_{S}$ and the mapping sequence $<m_{2}, m_{3}>$. Figure 3(e) shows the by-tuple answers of $Q$ with respect to $D_{S}$ and $p M$. 


\section{Complexity of Query Answering}

This section considers query answering in the presence of probabilistic mappings. We describe algorithms for query answering and study the complexity of query answering in terms of the size of the data (data complexity) and the size of the mapping (mapping complexity). We also consider cases in which we are not interested in the actual probability of an answer, just whether or not a tuple is a possible answer.

We show that when the schema is fixed, returning all by-table answers is in PTIME for both complexity measures, whereas returning all by-tuple answers in general is \#P-complete with respect to the data complexity. Recall that \#P is the complexity class of some hard counting problems (e.g., counting the number of variable assignments that satisfy a boolean formula). It is believed that a \#P-complete problem cannot be solved in polynomial time, unless $P=N P$. We show that computing the probabilities is the culprit here: even deciding the probability of a single answer tuple under by-tuple semantics is already \#P-complete, whereas computing all by-tuple answers without returning the probabilities is in PTIME. Finally, we identify a large subclass of common queries where returning all by-tuple answers with their probabilities is still in PTIME.

\subsection{By-table Query Answering}

In the case of by-table semantics, answering queries is conceptually simple. Given a p-mapping $p M=(S, T, \mathbf{m})$ and an SPJ query $Q$, we can compute the certain answers of $Q$ under each of the mappings $m \in \mathbf{m}$. We attach the probability $\operatorname{Pr}(m)$ to every certain answer under $m$. If a tuple is an answer to $Q$ under multiple mappings in $\mathbf{m}$, then we add up the probabilities of the different mappings.

Algorithm ByTABLE takes as input an SPJ query $Q$ that mentions the relations $T_{1}, \ldots, T_{l}$ in the FROM clause. Assume that we have the p-mapping $p M_{i}$ associated with the table $T_{i}$. The algorithm proceeds as follows.

Step 1: We generate the possible reformulations of $Q$ (a reformulation query computes all certain answers when executed on the source data) by considering every combination of the form $\left(m^{1}, \ldots, m^{l}\right)$, where $m^{i}$ is one of the possible mappings in $p M_{i}$. Denote the set of reformulations by $Q_{1}^{\prime}, \ldots, Q_{k}^{\prime}$. The probability of a reformulation $Q^{\prime}=\left(m^{1}, \ldots, m^{l}\right)$ is $\Pi_{i=1}^{l} \operatorname{Pr}\left(m^{i}\right)$.

Step 2: For each reformulation $Q^{\prime}$, retrieve each of the unique answers from the sources. For each answer obtained by $Q_{1}^{\prime} \cup \ldots \cup Q_{k}^{\prime}$, its probability is computed by summing the probabilities of the $Q^{\prime}$ 's in which it is returned.

Importantly, note that it is possible to express both steps as a SQL query with grouping and aggregation. Therefore, if the underlying sources support SQL, we can leverage their optimizations to compute the answers.

With our restricted form of schema mapping, the algorithm takes time polynomial in the size of the data and the mappings. We thus have the following complexity result. We give full proofs for results in this paper in the Appendix.

Theorem 4.1. Let $\overline{p M}$ be a schema p-mapping and let $Q$ be an SPJ query.

Answering $Q$ with respect to $\overline{p M}$ in by-table semantics is in PTIME in the size of the data and the mapping.

GLAV mappings: It is rather straightforward to extend the above results to arbitrary GLAV mappings. We define general p-mappings to be triples of the form $p G M=(\bar{S}, \bar{T}$, gm), where gm 


\begin{tabular}{c|c}
\hline Tuple & Prob \\
\hline ('Sunnyvale') & 0.94 \\
('Mountain View') & 0.5 \\
('alice@') & 0.1 \\
('bob@') & 0.1 \\
\hline
\end{tabular}

(a)

\begin{tabular}{c|c}
\hline Tuple & Prob \\
\hline ('Sunnyvale') & 0.8 \\
('Mountain View') & 0.8 \\
\hline
\end{tabular}

(b)

Figure 4: Example 4.3: (a) $Q_{1}^{\text {tuple }}(D)$ and (b) $Q_{2}^{\text {tuple }}(D)$.

is a set $\left\{\left(g m_{i}, \operatorname{Pr}\left(g m_{i}\right)\right) \mid i \in[1, n]\right\}$, such that for each $i \in[1, n], g m_{i}$ is a general GLAV mapping. The definition of by-table semantics for such mappings is a simple generalization of Definition 3.7. The following result holds for general p-mappings.

Theorem 4.2. Let $p G M$ be a general p-mapping between a source schema $\bar{S}$ and a target schema $\bar{T}$. Let $D_{S}$ be an instance of $\bar{S}$. Let $Q$ be an SPJ query with only equality conditions over $\bar{T}$. The problem of computing $Q^{\text {table }}\left(D_{S}\right)$ with respect to $p G M$ is in PTIME in the size of the data and the mapping.

\subsection{By-tuple Query Answering}

To extend the by-table query-answering strategy to by-tuple semantics, we would need to compute the certain answers for every mapping sequence generated by $p M$. However, the number of such mapping sequences is exponential in the size of the input data. The following example shows that for certain queries this exponential time complexity is not avoidable.

Example 4.3. Suppose that in addition to the tables in Example 2.1, we also have U(city) in the source and $\mathrm{V}$ (hightech) in the target. The p-mapping for $V$ contains two possible mappings: (\{ (city, hightech)\}, .8) and ( $\emptyset, .2)$.

Consider the following query $Q$, which decides if there are any people living in a high-tech city.

Q: SELECT 'true'

FROM T, V

WHERE T.mailing-addr $=$ V.hightech

One may conjecture that we can answer the query by first executing the following two sub-queries $Q_{1}$ and $Q_{2}$, then joining the answers of $Q_{1}$ and $Q_{2}$ and summing up the probabilities.

\section{Q1: SELECT mailing-addr FROM $T$}

Q2: SELECT hightech FROM V

Now consider the source instance $D$, where $D_{S}$ is shown in Figure 2(a), and $D_{U}$ has two tuples ('Mountain View') and ('Sunnyvale'). Figure $4(a)$ and (b) show $Q_{1}^{\text {tuple }}(D)$ and $Q_{2}^{\text {tuple }}(D)$. If we join the results of $Q_{1}$ and $Q_{2}$, we obtain for the true tuple the following probability: $0.94 * 0.8+0.5 * 0.8=$ 1.152. However, this is incorrect. By enumerating all consistent target tables, we in fact compute 0.864 as the probability. The reason for this error is that on some target instance that is by-tuple consistent with the source instance, the answers to both $Q_{1}$ and $Q_{2}$ contain tuple ('Sunnyvale') and tuple ('Mountain View'). Thus, generating the tuple ('Sunnyvale') as an answer for both $Q_{1}$ and $Q_{2}$ and generating the tuple ('Mountain View') for both queries are not independent events, so simply adding up their probabilities leads to incorrect results.

Indeed, we cannot answer $Q$ by dividing it into several sub-queries and then joining the results in some way, but have to answer the query by enumerating all by-tuple consistent target instances. 
In fact, we show that in general, answering SPJ queries in by-tuple semantics with respect to schema p-mappings is hard.

Theorem 4.4. Let $Q$ be an SPJ query and let $\overline{p M}$ be a schema p-mapping. The problem of finding the probability for a by-tuple answer to $Q$ with respect to $\overline{p M}$ is \#P-complete with respect to data complexity and is in PTIME with respect to mapping complexity.

The lower bound in Theorem 4.4 is proved by reducing the problem of counting the number of variable assignments that satisfy a bipartite monotone $2 \mathrm{DNF}$ boolean formula to the problem of finding the answers to $Q$. We give the full proof of this theorem in Appendix 12 .

In fact, the reason for the high complexity is exactly that we are asking for the probability of the answer. The following theorem shows that if we only want to know the possible by-tuple answers, we can do so in polynomial time.

Theorem 4.5. Given an SPJ query and a schema p-mapping, returning all by-tuple answers without probabilities is in PTIME with respect to data complexity.

The key to proving the PTIME complexity is that we can find all by-tuple answer tuples (without knowing the probability) by answering the query on the mirror target of the source data. Formally, let $D_{S}$ be the source data and $\overline{p M}$ be the schema p-mapping. The mirror target of $D_{S}$ with respect to $\overline{p M}$ is defined as follows. If $R$ is not involved in any mapping, the mirror target contains $R$ itself; if $R$ is the target of $p M=(S, T, \mathbf{m}) \in \overline{p M}$, the mirror target contains a relation $R^{\prime}$ where for each source tuple $t_{S}$ of $S$ and each $m \in \mathbf{m}$, there is a tuple $t_{T}$ in $R^{\prime}$ that (1) is consistent with $t_{S}$ and $m$ and contains null value for each attribute that is not involved in $m,(2)$ contains an id column with the value of the id column in $t_{S}$ (we assume the existence of identifier attribute id for $S$ and in practice we can use $S$ 's key attributes in place of id), and (3) contains a mapping column with the identifier of $m$. Meanwhile, we slightly modify a query $Q$ into a mirror query $Q_{m}$ with respect to $\overline{p M}$ as follows: $Q_{m}$ is the same as $Q$ except that for each relation $R$ that is the target of a p-mapping in $\overline{p M}$ and occurs multiple times in $Q$ 's FROM clause, and for any of $R$ 's two aliases $R_{1}$ and $R_{2}$ in the FROM clause, $Q^{\prime}$ contains in addition the following predicates: $\left(R_{1}\right.$.id $<>R_{2}$.id $\mathrm{OR} R_{1}$.mapping $=R_{2}$.mapping).

Lemma 4.6. Let $\overline{p M}$ be a schema p-mapping. Let $Q$ be an SPJ query and $Q_{m}$ be $Q$ 's mirror query with respect to $\overline{p M}$. Let $D_{S}$ be the source database and $D_{T}$ be the mirror target of $D_{S}$ with respect to $\overline{p M}$.

Then, $t \in Q^{\text {tuple }}\left(D_{S}\right)$ if and only if $t \in Q_{m}\left(D_{T}\right)$ and $t$ does not contain null value.

The size of the mirror target is polynomial in the size of the data and the p-mapping. The PTIME complexity bound follows from the fact that answering the mirror query on the mirror target takes only polynomial time.

GLAV mappings: Extending by-tuple semantics to arbitrary GLAV mappings is much trickier than by-table semantics. It would involve considering mapping sequences whose length is the product of the number of tuples in each source table, and the results are much less intuitive. Hence, we postpone by-tuple semantics to future work.

\subsection{Two Restricted Cases}

In this section we identify two restricted but common classes of queries for which by-tuple query answering takes polynomial time. We conjecture that they are the only cases where it is possible to answer a query in polynomial time. 
In our discussion we refer to subgoals of a query. The subgoals are tables that occur in the FROM clause of a query. Hence, even if the same table occurs twice in the FROM clause, each occurrence is a different subgoal.

\section{Queries with a single p-mapping subgoal}

The first class of queries we consider are those that include only a single subgoal being the target of a p-mapping. Relations in the other subgoals are either involved in ordinary mappings or do not require a mapping. Hence, if we only have uncertainty with respect to one part of the domain, our queries will typically fall in this class. We call such queries non-p-join queries. The query $Q$ in the motivating example is an example non-p-join query.

Definition 4.7 (non-p-join queries). Let $\overline{p M}$ be a schema p-mapping and let $Q$ be an SPJ query.

If at most one subgoal in the body of $Q$ is the target of a p-mapping in $\overline{p M}$, then we say $Q$ is a non-p-join query with respect to $\overline{p M}$.

For a non-p-join query $Q$, the by-tuple answers of $Q$ can be generated from the by-table answers of $Q$ over a set of databases, each containing a single tuple in the source table. Specifically, let $p M=(S, T, \mathbf{m})$ be the single p-mapping whose target is a relation in $Q$, and let $D_{S}$ be an instance of $S$ with $d$ tuples. Consider the set of tuple databases $\mathbf{T}\left(D_{S}\right)=\left\{D_{1}, \ldots, D_{d}\right\}$, where for each $i \in[1, d], D_{i}$ is an instance of $S$ and contains only the $i$-th tuple in $D_{S}$. The following lemma shows that $Q^{\text {tuple }}\left(D_{S}\right)$ can be derived from $Q^{\text {table }}\left(D_{1}\right), \ldots, Q^{\text {table }}\left(D_{d}\right)$.

Lemma 4.8. Let $\overline{p M}$ be a schema p-mapping between $\bar{S}$ and $\bar{T}$. Let $Q$ be a non-p-join query over $\bar{T}$ and let $D_{S}$ be an instance of $\bar{S}$. Let $(t, \operatorname{Pr}(t))$ be a by-tuple answer with respect to $D_{S}$ and $\overline{p M}$. Let $\bar{T}(t)$ be the subset of $\mathbf{T}\left(D_{S}\right)$ such that for each $D \in \bar{T}(t), t \in Q^{\text {table }}(D)$. The following two conditions hold:

1. $\bar{T}(t) \neq \emptyset ;$

2. $\operatorname{Pr}(t)=1-\Pi_{D \in \bar{T}(t),(t, p) \in Q^{\text {table }}(D)}(1-p)$.

In practice, answering the query for each tuple database can be expensive. We next describe Algorithm NonPJoin, which computes the answers for all tuple databases in one step. The key of the algorithm is to distinguish answers generated by different source tuples. To do this, we assume there is an identifier attribute id for the source relation whose values are concatenations of values of the key columns. We now describe the algorithm in detail.

Algorithm NonPJoIn takes as input a non-p-join query $Q$, a schema p-mapping $\overline{p M}$, and a source instance $D_{S}$, and proceeds in three steps to compute all by-tuple answers.

Step 1: Rewrite $Q$ to $Q^{\prime}$ such that it returns T.id in addition. Revise the p-mapping such that each possible mapping contains the correspondence between $S$.id and T.id.

Step 2: Invoke ByTABLE with $Q^{\prime}, \overline{p M}$ and $D_{S}$. Note that each generated result tuple contains the id column in addition to the attributes returned by $Q$.

Step 3: Project the answers returned in Step 2 on $Q$ 's returned attributes. Suppose projecting $t_{1}, \ldots, t_{n}$ obtains the answer tuple $t$, then the probability of $t$ is $1-\Pi_{i=1}^{n}\left(1-\operatorname{Pr}\left(t_{i}\right)\right)$.

Example 4.9. Consider rewriting $Q$ in the motivating example, repeated as follows:

\section{Q: SELECT mailing-addr FROM $\mathrm{T}$}


Step 1 rewrites $Q$ into query $Q^{\prime}$ by adding the id column:

Q': SELECT id, mailing-addr FROM $\mathrm{T}$ $Q^{\prime}$ :

In Step 2, BYTABLE may generate the following SQL query to compute by-table answers for

Qa: SELECT id, mailing-addr, SUM(pr)

FROM (

SELECT DISTINCT id, current-addr AS mailing-addr, $0.5 \mathrm{AS}$ pr

FROM S

UNION ALL

SELECT DISTINCT id, permanent-addr AS mailing-addr, 0.4 AS pr

FROM S

UNION ALL

SELECT DISTINCT id, email-addr AS mailing-addr, 0.1 AS pr

FROM S)

GROUP BY id, mailing-addr

Step 3 then generates the results using the following query.

Qu: SELECT mailing-addr, NOR(pr) AS pr

FROM Qa

GROUP BY mailing-addr

where for a set of probabilities $p r_{1}, \ldots, p r_{n}$, NOR computes $1-\Pi_{i=1}^{n} p r_{i}$.

An analysis of Algorithm NonPJoIN leads to the following complexity result for non-p-join queries.

Theorem 4.10. Let $\overline{p M}$ be a schema p-mapping and let $Q$ be a non-p-join query with respect to $\overline{p M}$.

Answering $Q$ with respect to $\overline{p M}$ in by-tuple semantics is in PTIME in the size of the data and the mapping.

\section{Projected p-join queries}

We now show that query answering can be done in polynomial time for a class of queries, called projected p-join queries, that include multiple subgoals involved in p-mappings. In such a query, we say that a join predicate is a $p$-join predicate with respect to a schema $\mathrm{p}$-mapping $\overline{p M}$, if at least one of the involved relations is the target of a p-mapping in $\overline{p M}$. We define projected p-join queries as follows.

Definition 4.11 (projected p-join query). Let $\overline{p M}$ be a schema p-mapping and $Q$ be an SPJ query over the target of $\overline{p M}$. If the following conditions hold, we say $Q$ is a projected p-join query with respect to $\overline{p M}$ :

- at least two subgoals in the body of $Q$ are targets of p-mappings in $\overline{p M}$.

- for every p-join predicate, the join attribute (or an equivalent attribute implied by the predicates in $Q$ ) is returned in the SELECT clause. 
Example 4.12. Consider the schema p-mapping in Example 4.3. A slight revision of $Q$, shown as follows, is a non-p-join query.

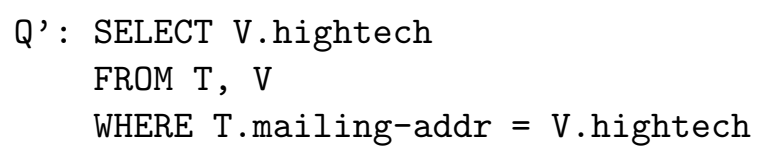

Note that in practice, when joining data from multiple tables in a data integration scenario, we typically project the join attributes, thereby leading to projected p-join queries.

The key to answering a projected-p-join query $Q$ is to divide $Q$ into multiple subqueries, each of which is a non-p-join query, and compute the answer to $Q$ from the answers to the subqueries. We proceed by considering partitions of the subgoals in $Q$. We say that a partitioning $\bar{J}$ is a refinement of a partitioning $\bar{J}^{\prime}$, denoted $\bar{J} \preceq \bar{J}^{\prime}$, if for each partition $J \in \bar{J}$, there is a partition $J^{\prime} \in \bar{J}^{\prime}$, such that $J \subseteq J^{\prime}$. We consider the following partitioning of $Q$, the generation of which will be described in detail in the algorithm.

Definition 4.13 (Maximal P-Join Partitioning). Let $\overline{p M}$ be a schema p-mapping. Let $Q$ be an $S P J$ query and $\bar{J}$ be a partitioning of the subgoals in $Q$.

We say that $\bar{J}$ is a p-join partitioning of $Q$, if (1) each partition $J \in \bar{J}$ contains at most one subgoal that is the target of a p-mapping in $\overline{p M}$, and (2) if neither subgoal in a join predicate is involved in p-mappings in $\overline{p M}$, the two subgoals belong to the same partition.

We say that $\bar{J}$ is a maximal p-join partitioning of $Q$, if there does not exist a p-join partitioning $\bar{J}^{\prime}$, such that $\bar{J} \preceq \bar{J}^{\prime}$.

For each partition $J \in \bar{J}$, we can define a query $Q_{J}$ as follows. The FROM clause includes the subgoals in $J$. The SELECT clause includes $J$ 's attributes that occur in (1) $Q$ 's SELECT clause or (2) $Q$ 's join predicates that join subgoals in $J$ with subgoals in other partitions. The WHERE clause includes $Q$ 's predicates that contain only subgoals in $J$. When $J$ is a partition in a maximal p-join partitioning of $Q$, we say that $Q_{J}$ is a p-join component of $Q$.

The following is the main lemma underlying our algorithm. It shows that we can compute the answers of $Q$ from the answers to its p-join components.

Lemma 4.14. Let $\overline{p M}$ be a schema p-mapping. Let $Q$ be a projected p-join query with respect to $\overline{p M}$ and let $\bar{J}$ be a maximal p-join partitioning of $Q$. Let $Q_{J 1}, \ldots, Q_{J n}$ be the p-join components of $Q$ with respect to $\bar{J}$.

For any instance $D_{S}$ of the source schema of $\overline{p M}$ and result tuple $t \in Q^{\text {tuple }}\left(D_{S}\right)$, the following two conditions hold:

1. For each $i \in[1, n]$, there exists a single tuple $t_{i} \in Q_{J i}^{\text {tuple }}\left(D_{S}\right)$, such that $t_{1}, \ldots, t_{n}$ generate $t$ when joined together.

2. Let $t_{1}, \ldots, t_{n}$ be the above tuples. Then $\operatorname{Pr}(t)=\Pi_{i=1}^{n} \operatorname{Pr}\left(t_{i}\right)$.

Lemma 4.14 leads naturally to the query-rewriting algorithm ProJeCTEDPJoIn, which takes as input a projected-p-join query $Q$, a schema p-mapping $\overline{p M}$, and a source instance $D_{S}$, outputs all by-tuple answers, and proceeds in three steps.

Step 1: Generate maximum p-join partitions $J_{1}, \ldots, J_{n}$ as follows. First, initialize each partition to contain one subgoal in $Q$. Then, for each join predicate with subgoals $S_{1}$ and $S_{2}$ that are not 
involved in p-mappings in $\overline{p M}$, merge the partitions that $S_{1}$ and $S_{2}$ belong to. Finally, for each partition that contains no subgoal involved in $\overline{p M}$, merge it with another partition.

Step 2: For each p-join partition $J_{i}, i \in[1, n]$, generate the p-join component $Q_{J i}$ and invoke Algorithm NonPJoIn with $Q_{J i}, \overline{p M}$ and $D_{S}$ to compute answers for $Q_{J i}$.

Step 3: Join the results of $Q_{J 1}, \ldots, Q_{J n}$. If an answer tuple $t$ is obtained by joining $t_{1}, \ldots, t_{n}$, then the probability of $t$ is computed by $\prod_{i=1}^{n} \operatorname{Pr}\left(t_{i}\right)$.

We illustrate the algorithm using the following example.

Example 4.15. Consider query $Q^{\prime}$ in Example 4.12. Its two p-join components are $Q_{1}$ and $Q_{2}$ shown in Example 4.3. Suppose we compute $Q_{1}$ with query $Q_{u}$ (shown in Example 4.9) and compute $Q_{2}$ with query $Q_{u}^{\prime}$. We can compute by-tuple answers of $Q^{\prime}$ as follows:

SELECT Qu'.hightech, Qu.pr*Qu'.pr

FROM Qu, Qu'

WHERE Qu.mailing-addr $=$ Qu'.hightect

Since the number of p-join components is bounded by the number of subgoals in a query, and for each of them we invoke Algorithm NonPJoin, query answering for projected p-join queries takes polynomial time.

Theorem 4.16. Let $\overline{p M}$ be a schema p-mapping and let $Q$ be a projected-p-join query with respect to $\overline{p M}$.

Answering $Q$ with respect to $\overline{p M}$ in by-tuple semantics is in PTIME in the size of the data and the mapping.

\section{Other SPJ queries}

A natural question is whether the two classes of queries we have identified are the only ones for which query answering is in PTIME for by-tuple semantics. As Example 4.3 shows, if $Q$ contains multiple subgoals that are involved in a schema p-mapping, but $Q$ is not a projected-pjoin query, then Condition 1 in Lemma 4.14 does not hold and query answering needs to proceed by enumerating all mapping sequences.

We believe that the complexity of the border case, where a query joins two relations involved in p-mappings but does not return the join attribute, is \#P-hard, but currently it remains an open problem.

\section{$5 \quad$ Top- $K$ Query Answering}

In this section, we consider returning the top- $k$ query answers, which are the $k$ answer tuples with the top probabilities. The main challenge in designing the algorithm is to only perform the necessary reformulations at every step and halt when the top- $k$ answers are found. We first describe our algorithm for by-table semantics. We then show the challenges for by-tuple semantics and outline our solution. 


\subsection{Returning Top- $K$ By-table Answers}

Recall that in by-table query answering, the probability of an answer is the sum of the probabilities of the reformulated queries that generate the answer. Our goal is to reduce the number of reformulated queries we execute. Our algorithm proceeds in a greedy fashion: we execute queries in descending order of probabilities. For each tuple $t$, we maintain the upper bound $p_{\max }(t)$ and lower bound $p_{\min }(t)$ of its probability. This process halts when we find $k$ tuples whose $p_{\min }$ values are higher than $p_{\max }$ of the rest of the tuples.

TOPKBYTABLE takes as input an SPJ query $Q$, a schema p-mapping $\overline{p M}$, an instance $D_{S}$ of the source schema, and an integer $k$, and outputs the top- $k$ answers in $Q^{\text {table }}\left(D_{S}\right)$. The algorithm proceeds in three steps.

Step 1: Rewrite $Q$ according to $\overline{p M}$ into a set of queries $Q_{1}, \ldots, Q_{n}$, each with a probability assigned in a similar way as stated in Algorithm ByTABLE.

Step 2: Execute $Q_{1}, \ldots, Q_{n}$ in descending order of their probabilities. Maintain the following measures:

- The highest probability, PMax, for the tuples that have not been generated yet. We initialize $P M a x$ to 1; after executing query $Q_{i}$ and updating the list of answers (see third bullet), we decrease $\operatorname{PMax}$ by $\operatorname{Pr}\left(Q_{i}\right)$;

- The threshold th determining which answers are potentially in the top- $k$. We initialize $t h$ to 0 ; after executing $Q_{i}$ and updating the answer list, we set th to the $k$-th largest $p_{\min }$ for tuples in the answer list;

- A list $L$ of answers whose $p_{\max }$ is no less than $t h$, and bounds $p_{\min }$ and $p_{\max }$ for each answer in $L$. After executing query $Q_{i}$, we update the list as follows: (1) for each $t \in L$ and $t \in Q_{i}\left(D_{S}\right)$, we increase $p_{\min }(t)$ by $\operatorname{Pr}\left(Q_{i}\right)$; (2) for each $t \in L$ but $t \notin Q_{i}\left(D_{S}\right)$, we decrease $p_{\max }(t)$ by $\operatorname{Pr}\left(Q_{i}\right) ;(3)$ if $P M a x \geq t h$, for each $t \notin L$ but $t \in Q_{i}\left(D_{S}\right)$, insert $t$ to $L$, set $p_{\text {min }}$ to $\operatorname{Pr}\left(Q_{i}\right)$ and $p_{\max }(t)$ to $P M a x$.

- A list $T$ of $k$ tuples with top $p_{\min }$ values.

Step 3: When $t h>P M a x$ and for each $t \notin T, t h>p_{\max }(t)$, halt and return $T$.

Example 5.1. Consider Example 2.1 where we seek for top-1 answer. We answer the reformulated queries in order of $Q_{1}, Q_{2}, Q_{3}$. After answering $Q_{1}$, for tuple ("Sunnyvale") we have $p_{\min }=.5$ and $p_{\max }=1$, and for tuple ("Mountain View") we have the same bounds. In addition, PMax $=.5$ and $t h=.5$.

In the second round, we answer $Q_{2}$. Then, for tuple ("Sunnyvale") we have $p_{\min }=.9$ and $p_{\max }=1$, and for tuple ("Mountain View") we have $p_{\min }=.5$ and $p_{\max }=.6$. Now PMax $=.1$ and $t h=.9$.

Because th > PMax and th is above the $p_{\max }$ for the ("Mountain View") tuple, we can halt and return ("Sunnyvale") as the top-1 answer.

The next theorem states the correctness of ByTableTopK.

Theorem 5.2. For any schema mapping $\overline{p M}$, SPJ query $Q$, instance $D_{S}$ of the source schema of $\overline{p M}$, and integer $k$, Algorithm BYTABLETOPK correctly computes the top- $k$ answers in $Q^{\text {table }}\left(D_{S}\right)$.

Our algorithm differs from previous top- $k$ algorithms in the literature in two aspects. First, we execute the reformulated queries only when necessary, so we can return the top- $k$ answers without 
executing all reformulated queries thereby leading to significant performance improvements. Fagin et al. [9] have proposed several algorithms for finding instances with top- $k$ scores, where each instance has $m$ attributes and the score of the instance is an aggregation over values of these $m$ attributes. However, these algorithms assume for each attribute there exists a sorted list on its values, and they access the lists in parallel. In our context, this would require executing all reformulated queries upfront. Li et al. [19] have studied computing top- $k$ answers for aggregation and group-by queries and optimizing query answering by generating the groups incrementally. Although we can also compute by-table answers using an aggregation query, this query is different from those considered in [19] in that the WHERE clause contains a set of sub-queries rather than database tables. Therefore, applying [19] here also requires evaluating all reformulated queries at the beginning.

Second, whereas maintaining upper bounds and lower bounds for instances has been explored in the literature, such as in Fagin's NRA (Non-Random Access) algorithm and in [19], our algorithm is different in that it keeps these bounds only for tuples that have already been generated by an executed reformulated query and that are potential top- $k$ answers (by judging if the upper bound is above the threshold $t h$ ). In addition, we are different from [19] in that we do not assume the threshold $t h$ is known beforehand.

\subsection{By-tuple Top- $K$ Query Answering}

We next consider returning top- $k$ answers in by-tuple semantics. In general, we need to consider each mapping consequence and answer the query on the target instance that is consistent with the source and the mapping sequence. Algorithm TOPKByTABLE can be modified to compute top- $k$ by-tuple answers by deciding at runtime the mapping sequence to consider next. However, for non-p-join queries and projected-p-join queries, we can return top- $k$ answers more efficiently. We outline our method for answering non-p-join queries here, and for space considerations, we leave projected-p-join queries to the full paper.

For non-p-join queries the probability of an answer tuple $t$ to query $Q$ cannot be expressed as a function of $t$ 's probabilities in executing reformulations of $Q$; rather, it is a function of $t$ 's probabilities in answering $Q$ on each tuple database of the source table. However, retrieving answers on a tuple base is expensive. Algorithm NonPJorn provides a method that computes by-tuple answers on the tuple databases in a batch mode by first rewriting $Q$ into $Q^{\prime}$ by returning the id column and then executing $Q^{\prime}$ 's reformulated queries. We find top- $k$ answers in a similar fashion. Here, after executing each reformulated query, we need to maintain two answer lists, one for $Q$ and one for $Q^{\prime}$, and compute $p_{\min }$ and $p_{\max }$ for answers in different lists differently.

\section{Representation of Probabilistic Mappings}

Thus far, a p-mapping was represented by listing each of its possible mappings, and the complexity of query answering was polynomial in the size of that representation. Such a representation can be quite lengthy since it essentially enumerates a probability distribution by listing every combination of events in the probability space. Hence, an interesting question is whether there are more concise representations of p-mappings and whether our algorithms can leverage them.

We consider three representations that can reduce the size of the p-mapping exponentially. In Section 6.1 we consider a representation in which the attributes of the source and target tables are partitioned into groups and p-mappings are specified for each group separately. We show that query answering can be done in time polynomial in the size of the representation. In Section 6.2 we 


\begin{tabular}{c|c}
\hline Mapping & Prob \\
\hline$\left\{\left(\mathrm{a}, \mathrm{a}^{\prime}\right),\left(\mathrm{b}, \mathrm{b}^{\prime}\right),\left(\mathrm{c}, \mathrm{c}^{\prime}\right)\right\}$ & 0.72 \\
$\left\{\left(\mathrm{a}, \mathrm{b}^{\prime}\right),\left(\mathrm{c}, \mathrm{c}^{\prime}\right)\right\}$ & 0.18 \\
$\left\{\left(\mathrm{a}, \mathrm{a}^{\prime}\right),\left(\mathrm{b}^{\prime} \mathrm{b}^{\prime}\right)\right\}$ & 0.08 \\
$\left\{\left(\mathrm{a}, \mathrm{b}^{\prime}\right)\right\}$ & 0.02 \\
\hline
\end{tabular}

(a)

\begin{tabular}{c|c}
\hline Mapping & Prob \\
\hline$\left\{\left(\mathrm{a}, \mathrm{a}^{\prime}\right),\left(\mathrm{b}, \mathrm{b}^{\prime}\right)\right\}$ & 0.8 \\
$\left\{\left(\mathrm{a}, \mathrm{b}^{\prime}\right)\right\}$ & 0.2 \\
\hline
\end{tabular}

(b)

\begin{tabular}{c|c}
\hline Mapping & Prob \\
\hline$\left\{\left(\mathrm{c}, \mathrm{c}^{\prime}\right)\right\}$ & 0.9 \\
$\emptyset$ & 0.1 \\
\hline
\end{tabular}

(c)

Figure 5: Example 6.2: the p-mapping in (a) is equivalent to the 2-group p-mapping in (b) and (c).

consider probabilistic correspondences, where we specify the marginal probability of each attribute correspondence. However, we show that such a representation can only be leveraged in limited cases. Finally, we consider Bayes Nets, the most common method for concisely representing probability distributions, in Section 6.3, and show that even though some p-mappings can be represented by them, query answering does not necessarily benefit from the representation.

\subsection{Group Probabilistic Mapping}

In practice, the uncertainty we have about a p-mapping can often be represented as a few localized choices, especially when schema mappings are created by semi-automatic methods. To represent such p-mappings more concisely, we can partition the source and target attributes and specify p-mappings for each partition.

Definition 6.1 (Group P-Mapping). An n-group p-mapping gp $M$ is a triple $(S, T, \overline{p M})$, where

- $S$ is a source relation schema and $S_{1}, \ldots, S_{n}$ is a set of disjoint subsets of attributes in $S$;

- $T$ is a target relation schema and $T_{1}, \ldots, T_{n}$ is a set of disjoint subsets of attributes in $T$;

- $\overline{p M}$ is a set of $p$-mappings $\left\{p M_{1}, \ldots, p M_{n}\right\}$, where for each $1 \leq i \leq n, p M_{i}$ is a p-mapping between $S_{i}$ and $T_{i}$.

The semantics of an $n$-group p-mapping $g p M=(S, T, \overline{p M})$ is a p-mapping that includes the Cartesian product of the mappings in each of the $p M_{i}$ 's. The probability of the mapping composed of $m_{1} \in p M_{1}, \ldots, m_{n} \in p M_{n}$ is $\prod_{i=1}^{n} \operatorname{Pr}\left(m_{i}\right)$.

Example 6.2. Figure 5(a) shows p-mapping $p M$ between the schemas $S(a, b, c)$ and $T\left(a^{\prime}, b^{\prime}, c^{\prime}\right)$. Figure 5(b) and (c) show two independent mappings that together form a 2-group p-mapping equivalent to $p M$.

Note that a group p-mapping can be considerably more compact than an equivalent p-mapping. Specifically, if each $p M_{i}$ includes $l_{i}$ mappings, then a group p-mapping can describe $\Pi_{i=1}^{n} l_{i}$ possible mappings with $\sum_{i=1}^{n} l_{i}$ sub-mappings. The important feature of $n$-group p-mappings is that query answering can be done in time polynomial in their size.

Theorem 6.3. Let $\overline{g p M}$ be a schema group p-mapping and let $Q$ be an SPJ query. The mapping complexity of answering $Q$ with respect to $\overline{g p M}$ in both by-table semantics and by-tuple semantics is in PTIME.

Note that as $n$ grows, fewer p-mappings can be represented with $n$-group p-mappings. Formally, suppose we denote by $\mathcal{M}_{S T}^{n}$ the set of all $n$-group p-mappings between $S$ and $T$, then: 
Proposition 6.4. For each $n \geq 1, \mathcal{M}_{S T}^{n+1} \subset \mathcal{M}_{S T}^{n}$.

We typically expect that when possible, a mapping would be given as a group p-mapping. The following theorem shows that we can find the best group p-mapping for a given p-mapping in polynomial time.

Theorem 6.5. Given a p-mapping $p M$, we can find in polynomial time in the size of $p M$ the maximal $n$ and an n-group p-mapping gp $M$, such that gpM is equivalent to $p M$.

\subsection{Probabilistic Correspondences}

The second representation we consider, probabilistic correspondences, represents a p-mapping with the marginal probabilities of attribute correspondences. This representation is the most compact one as its size is proportional to the product of the schema sizes of $S$ and $T$.

Definition 6.6 (Probabilistic Correspondences). A probabilistic correspondence mapping (p-correspondence) is a triple $p C=(S, T, \mathbf{c})$, where $S=\left\langle s_{1}, \ldots, s_{m}\right\rangle$ is a source relation schema, $T=\left\langle t_{1}, \ldots, t_{n}\right\rangle$ is a target relation schema, and

- c is a set $\left\{\left(c_{i j}, \operatorname{Pr}\left(c_{i j}\right)\right) \mid i \in[1, m], j \in[1, n]\right\}$, where $c_{i j}=\left(s_{i}, t_{j}\right)$ is an attribute correspondence, and $\operatorname{Pr}\left(c_{i j}\right) \in[0,1]$;

- for each $i \in[1, m], \sum_{j=1}^{n} \operatorname{Pr}\left(c_{i j}\right) \leq 1$;

- for each $j \in[1, n], \sum_{i=1}^{m} \operatorname{Pr}\left(c_{i j}\right) \leq 1$.

Note that for a source attribute $s_{i}$, we allow $\sum_{j=1}^{n} \operatorname{Pr}\left(c_{i j}\right)<1$. This is because in some of the possible mappings, $s_{i}$ may not be mapped to any target attribute. The same is true for target attributes.

From each p-mapping, we can infer a p-correspondence by calculating the marginal probabilities of each attribute correspondence. Specifically, for a p-mapping $p M=(S, T, \mathbf{m})$, we denote by $p C(p M)$ the p-correspondence where each marginal probability is computed as follows:

$$
\operatorname{Pr}\left(c_{i j}\right)=\sum_{c_{i j} \in m, m \in \mathbf{m}} \operatorname{Pr}(m)
$$

However, as the following example shows, the relationship between p-mappings and p-correspondences is many-to-one.

Example 6.7. The p-correspondence in Figure 6(b) is the one computed for both the p-mapping in Figure 6(a) and the p-mapping in Figure 5(a).

Given the many-to-one relationship, the question is when it is possible to compute the correct answer to a query based only on the p-correspondence. That is, we are looking for a class of queries $\bar{Q}$, called p-mapping independent queries, such that for every $Q \in \bar{Q}$ and every database instance $D_{S}$, if $p C\left(p M_{1}\right)=p C\left(p M_{2}\right)$, then the answer of $Q$ with respect to $p M_{1}$ and $D_{S}$ is the same as the answer of $Q$ with respect to $p M_{2}$ and $D_{S}$. Unfortunately, this property holds for a very restricted class of queries, defined as follows:

Definition 6.8 (Single-Attribute Query). Let $p C=(S, T, \mathbf{c})$ be a p-correspondence. An SPJ query $Q$ is said to be a single-attribute query with respect to $p C$ if $T$ has one single attribute occurring in the SELECT and WHERE clauses of $Q$. This attribute of $T$ is said to be a critical attribute. 
(a)

\begin{tabular}{c|c}
\hline Mapping & Prob \\
\hline$\left\{\left(\mathrm{a}, \mathrm{a}^{\prime}\right),\left(\mathrm{b}, \mathrm{b}^{\prime}\right),\left(\mathrm{c}, \mathrm{c}^{\prime}\right)\right\}$ & 0.8 \\
$\left\{\left(\mathrm{a}, \mathrm{b}^{\prime}\right),\left(\mathrm{c}, \mathrm{c}^{\prime}\right)\right\}$ & 0.1 \\
$\left\{\left(\mathrm{a}, \mathrm{b}^{\prime}\right)\right\}$ & 0.1 \\
\hline
\end{tabular}

\begin{tabular}{c|c}
\hline Corr & Prob \\
\hline$\left\{\left(\mathrm{a}, \mathrm{a}^{\prime}\right)\right\}$ & 0.8 \\
$\left\{\left(\mathrm{a}, \mathrm{b}^{\prime}\right)\right\}$ & 0.2 \\
$\left\{\left(\mathrm{~b}, \mathrm{~b}^{\prime}\right)\right\}$ & 0.8 \\
$\left\{\left(\mathrm{c}, \mathrm{c}^{\prime}\right)\right\}$ & 0.9 \\
\hline \multicolumn{2}{c}{$(\mathrm{b})$}
\end{tabular}

Figure 6: Example 6.7: the p-mapping in (a) corresponds to the p-correspondence in (b).

Theorem 6.9. Let $\overline{p C}$ be a schema p-correspondence, and $Q$ be an SPJ query. Then, $Q$ is $p$ mapping independent with respect to $\overline{p C}$ if and only if for each $p C \subseteq \overline{p C}, Q$ is a single-attribute query with respect to $p C$.

Example 6.10. Continuing with Example 6.7, consider the p-correspondence $p C$ in Figure 6(b) and the following two queries $Q_{1}$ and $Q_{2}$. Query $Q_{1}$ is mapping independent with respect to $p C$, but $Q_{2}$ is not.

\section{Q1: SELECT T.a FROM T,U WHERE T.a=U.a'}

Q2: SELECT T.a, T.c FROM T

Theorem 6.9 simplifies query answering for p-mapping independent queries. Wherever we needed to consider every possible mapping in previous algorithms, we consider only every attribute correspondence for the critical attribute.

Corollary 6.11. Let $\overline{p C}$ be a schema p-correspondence, and $Q$ be a p-mapping independent SPJ query with respect to $\overline{p C}$. The mapping complexity of answering $Q$ with respect to $\overline{p C}$ in both by-table semantics and by-tuple semantics is in PTIME.

The result in Theorem 6.9 can be generalized to cases where we know the p-mapping is an ngroup p-mapping. Specifically, as long as $Q$ includes at most a single attribute in each of the groups in the n-group p-mapping, query answering can still be done with the correspondence mapping. We omit the details of this generalization.

\subsection{Bayes Nets}

Bayes Nets are a powerful mechanism for concisely representing probability distributions and reasoning about probabilistic events [21]. The following example shows how Bayes Nets can be used in our context.

Example 6.12. Consider two schemas $S=\left(s_{1}, \ldots, s_{n}, s_{1}^{\prime}, \ldots, s_{n}^{\prime}\right)$ and $T=\left(t_{1}, \ldots, t_{n}\right)$. Consider the p-mapping $p M=(S, T, \mathbf{m})$, which describes the following probability distribution: if $s_{1}$ maps to $t_{1}$ then it is more likely that $\left\{s_{2}, \ldots, s_{n}\right\}$ maps to $\left\{t_{2}, \ldots, t_{n}\right\}$, whereas if $s_{1}^{\prime}$ maps to $t_{1}$ then it is more likely that $\left\{s_{2}^{\prime}, \ldots, s_{n}^{\prime}\right\}$ maps to $\left\{t_{2}, \ldots, t_{n}\right\}$.

We can represent the p-mapping using a Bayes Net as follows. Let $c$ be an integer constant. Then,

1. $\operatorname{Pr}\left(\left(s_{1}, t_{1}\right)\right)=\operatorname{Pr}\left(\left(s_{1}^{\prime}, t_{1}\right)\right)=1 / 2$;

2. for each $i \in[1, n], \operatorname{Pr}\left(\left(s_{i}, t_{i}\right) \mid\left(s_{1}, t_{1}\right)\right)=1-\frac{1}{c}$ and $\operatorname{Pr}\left(\left(s_{i}^{\prime}, t_{i}\right) \mid\left(s_{1}, t_{1}\right)\right)=\frac{1}{c}$; 
3. for each $i \in[1, n], \operatorname{Pr}\left(\left(s_{i}, t_{i}\right) \mid\left(s_{1}^{\prime}, t_{1}\right)\right)=\frac{1}{c}$ and $\operatorname{Pr}\left(\left(s_{i}^{\prime}, t_{i}\right) \mid\left(s_{1}^{\prime}, t_{1}\right)\right)=1-\frac{1}{c}$.

Since the p-mapping contains $2^{n}$ possible mappings, the original representation would take space $O\left(2^{n}\right)$; however, the Bayes-Net representation takes only space $O(n)$.

Although the Bayes-Net representation can reduce the size exponentially for some p-mappings, this conciseness may not help reduce the complexity of query answering. We formalize this result in the following theorem.

Theorem 6.13. There exists a schema p-mapping $\overline{p M}$ and a query $Q$, such that answering $Q$ with respect to $\overline{p M}$ in by-table semantics takes exponential time in the size of $\overline{p M}$ 's Bayes-Net representation.

\section{Probabilistic Data Exchange}

In this section we consider another method for using probabilistic schema mappings, and establish a close relationship between probabilistic mappings and probabilistic databases. We consider the scenario of data exchange, where data is shared by using the mappings to create an instance of the target schema called the core universal solution [8]. In our context, we show that we can create a probabilistic database that serves as the core universal solution.

Probabilistic databases: We begin by briefly reviewing probabilistic databases (the reader is referred to [24] for further details).

A probabilistic database (p-database) $p D$ over a schema $\bar{R}$ is a set $\left\{\left(D_{1}, \operatorname{Pr}\left(D_{1}\right)\right), \ldots,\left(D_{n}, \operatorname{Pr}\left(D_{n}\right)\right)\right\}$, such that

- for $i \in[1, n], D_{i}$ is an instance of $\bar{R}$, and for every $i, j \in[1, n], i \neq j \Rightarrow D_{i} \neq D_{j}$;

- $\operatorname{Pr}\left(D_{i}\right) \in[0,1]$ and $\sum_{i=1}^{n} \operatorname{Pr}\left(D_{i}\right)=1$.

Answers to queries over p-databases have probabilities associated with them. Specifically, let $Q$ be a query over $p D$, and let $t$ be a tuple. We denote by $\bar{D}(t)$ the subset of $p D$ such that for each $D \in \bar{D}(t), t \in Q(D)$. Let $p=\sum_{D \in \bar{D}(t)} \operatorname{Pr}(D)$. If $p>0$, we call $(t, p)$ a possible tuple in the answer of $Q$ on $p D$.

Given a query $Q$ and a p-database $p D$, we denote by $Q(p D)$ the set of all possible tuples in the answer of $Q$ on $p D$. We next show that data-exchange solutions can be represented as p-databases.

Data-exchange solutions: Informally, the data-exchange problem for a p-mapping $p M=(S, T, \mathbf{m})$ and an instance $D_{S}$ of $S$ is to find an instance of $T$ that is consistent with $D_{S}$ and $p M$. We distinguish between by-table solutions and by-tuple solutions.

Definition 7.1 (By-table Solution). Let $p M=(S, T, \mathbf{m})$ be a $p$-mapping and $D_{S}$ be an instance of $S$.

A p-database $p D_{T}=\left\{\left(D_{1}, \operatorname{Pr}\left(D_{1}\right)\right), \ldots,\left(D_{n}, \operatorname{Pr}\left(D_{n}\right)\right)\right\}$ is a by-table solution for $D_{S}$ under $p M$, if for each $i \in[1, n]$, there exists a subset $\overline{m_{i}} \subseteq \mathbf{m}$, such that

- for each $m \in \overline{m_{i}}, D_{i}$ is by-table consistent with $D_{S}$ and $m$;

- $\operatorname{Pr}\left(D_{i}\right)=\sum_{m \in \overline{m_{i}}} \operatorname{Pr}(m)$;

- $\bar{m}_{1}, \ldots, \bar{m}_{n}$ form a partition of $\mathbf{m}$. 
The intuition underlying the definition is that one target table $D_{i}$ may be consistent with a source table and a set of possible mappings $\bar{m}_{i}$, so the probability of $D_{i}$ is the sum of the probabilities of the mappings in $\bar{m}_{i}$. In the definition for by-tuple semantics, the same intuition applies, except that we need to consider subsets of sequences.

Definition 7.2 (By-tuple Solution). Let $p M=(S, T, \mathbf{m})$ be a $p$-mapping and $D_{S}$ be an instance of $S$ with d tuples.

A p-database $p D_{T}=\left\{\left(D_{1}, \operatorname{Pr}\left(D_{1}\right)\right), \ldots,\left(D_{n}, \operatorname{Pr}\left(D_{n}\right)\right)\right\}$ is a by-tuple solution for $D_{S}$ under $p M$ if for each $i \in[1, n]$, there exists a subset $\overline{s e q_{i}} \subseteq \mathbf{s e q}_{d}(p M)$, such that

- for each seq $\in \overline{s e q_{i}}, D_{i}$ is by-tuple consistent with $D_{S}$ and seq;

- $\operatorname{Pr}\left(D_{i}\right)=\sum_{s e q \in \overline{s e q}} \operatorname{Pr}(s e q)$;

- $\overline{s e q_{1}}, \ldots, \overline{s e q_{n}}$ form a partition of $\mathbf{s e q}_{d}(p M)$.

Core universal solution: Among all solutions, we would like to identify the core universal solution, because it is unique up to isomorphism and because we can use it to find all the answers to a query. We define the core universal solution for p-databases, but first we define homomorphisms on such databases.

Definition 7.3 (Homomorphism of P-Databases). Let $p D=\left\{\left(D_{i}, \operatorname{Pr}\left(D_{i}\right)\right) \mid i \in[1, n]\right\}$ and $p D^{\prime}=$ $\left\{\left(D_{i}^{\prime}, \operatorname{Pr}\left(D_{i}^{\prime}\right)\right)\right.$

$\mid i \in[1, m]\}$ be two p-databases of the same schema. Let $\mathcal{P}\left(p D^{\prime}\right)$ be the powerset of the databases in $p D^{\prime}$.

$A$ homomorphism $h: p D \rightarrow p D^{\prime}$ is a mapping from $p D$ to $\mathcal{P}\left(p D^{\prime}\right)$, such that

- for every $D \in p D$ and $D^{\prime} \in h(D)$, there exists a homomorphism $g: D \rightarrow D^{\prime}$;

- for every $D \in p D, \operatorname{Pr}(D)=\sum_{D^{\prime} \in h(D)} \operatorname{Pr}\left(D^{\prime}\right)$;

- $h\left(D_{1}\right), \ldots, h\left(D_{n}\right)$ form a partition of $p D^{\prime}$.

Note that in the above definition, a homomorphism can map a database in $p D$ to a set of databases in $p D^{\prime}$. We can now define core universal solutions.

Definition 7.4 (Core Universal Solution). Let $p M=(S, T, \mathbf{m})$ be a $p$-mapping and $D_{S}$ be an instance of $S$.

A $p$-database instance $p D_{T}$ of $T$ is called a by-table (resp. by-tuple) universal solution for $D_{S}$ under $p M$, if (1) $p D_{T}$ is a by-table (resp. by-tuple) solution for $D_{S}$, and (2) for every by-table (resp. by-tuple) solution $p D_{T}^{\prime}$ for $D_{S}$, there exists a homomorphism $h: p D_{T} \rightarrow p D_{T}^{\prime}$.

Further, $p D_{T}$ is called a by-table (resp. by-tuple) core universal solution for $D_{S}$ if for each possible database $D_{T} \in p D_{T}$, there is no homomorphism from $D_{T}$ to a proper subset of tuples in $D_{T}$.

The following theorem establishes the key properties of core universal solutions in our context.

Theorem 7.5. Let $p M=(S, T, \mathbf{m})$ be a p-mapping and $D_{S}$ be an instance of $S$.

1. There is a unique by-table (resp. by-tuple) core universal solution up to isomorphism for $D_{S}$ with respect to $\mathrm{pM}$.

2. Let $Q$ be a conjunctive query over $T$. Let $p D_{T}$ be the by-table (resp. by-tuple) core universal solution for $D_{S}$ under $p M$. Then,

$$
Q^{\text {table }}\left(D_{S}\right)=Q\left(p D_{T}\right) \quad\left(\text { resp. } Q^{\text {tuple }}\left(D_{S}\right)=Q\left(p D_{T}\right)\right)
$$


Complexity of data exchange: The complexity of computing the core universal solution is established by the following theorem:

Theorem 7.6. Let $p M=(S, T, \mathbf{m})$ be a p-mapping and $D_{S}$ be an instance of $S$.

Generating the by-table or by-tuple core universal solution for $D_{S}$ under $p M$ takes linear time in the size of the data and the mapping.

For by-table semantics the proof is rather straightforward. For by-tuple semantics the proof requires a special representation of p-databases, called disjunctive p-database.

Definition 7.7 (Disjunctive P-Database). Let $R$ be a relation schema where there exists a set of attributes that together form the key of the relation. Let $p D_{R}^{\vee}$ be a set of tuples of $R$, each attached with a probability.

We say that $p D_{R}^{\vee}$ is a disjunctive p-database if for each key value that occurs in $p D_{R}^{\vee}$, the probabilities of the tuples with this key value sum up to 1.

In a disjunctive p-database, we consider tuples with the same key value as disjoint and those with different key values as independent. Formally, let $k e y_{1}, \ldots, k e y_{n}$ be the set of all distinct key values in $p D_{R}^{\vee}$. For each $i \in[1, n]$, we denote by $d_{i}$ the number of tuples whose key value is $k e y_{i}$. Then, with a set of $\sum_{i=1}^{n} d_{i}$ tuples, $p D_{R}^{\vee}$ can define a set of $\prod_{i=1}^{n} d_{i}$ possible databases, where each possible database $(D, \operatorname{Pr}(D))$ contains $n$ tuples $t_{1}, \ldots, t_{n}$, such that $(1)$ for each $i \in[1, n]$, the key value of $t_{i}$ is $k e y_{i}$; and $(2) \operatorname{Pr}(D)=\Pi_{i=1}^{n} \operatorname{Pr}\left(t_{i}\right)$.

Theorem 7.6 is based on the following lemma.

Lemma 7.8. Let $p M=(S, T, \mathbf{m})$ be a p-mapping and $D_{S}$ be an instance of $S$.

The by-tuple core universal solution for $D_{S}$ under $p M$ can be represented as a disjunctive $p$ database.

The complexity of answering queries over the core universal solutions is the same as that of the corresponding results for probabilistic databases. Specifically, the following theorem follows from [23].

Theorem 7.9. Let $Q$ be a conjunctive query.

- Let $p D$ be a p-database instance. Computing $Q(p D)$ is in PTIME in the size of the data.

- Let $p D^{\vee}$ be a disjunctive p-database instance. Computing $Q\left(p D^{\vee}\right)$ is \#P-complete in the size of the data.

Finally, we note that when the p-mapping is a group p-mapping, we can compute the core universal solution in time that is linear in the size of the data and of the p-mapping.

\section{Broader Classes of Mappings}

In this section we briefly show how our results can be extended to capture two common practical extensions to our mapping language.

Complex mappings: Complex mappings map a set of attributes in the source to a set of attributes in the target. For example, we can map the attribute address to the concatenation of street, city, and state. 
Formally, a set correspondence between $S$ and $T$ is a relationship between a subset of attributes in $S$ and a subset of attributes in $T$. Here, the function associated with the relationship specifies a single value for each of the target attributes given a value for each of the source attributes. Again, the actual functions are irrelevant to our discussion. A complex mapping is a triple $(S, T, c m)$, where $\mathrm{cm}$ is a set of set correspondences, such that each attribute in $S$ or $T$ is involved in at most one set correspondence. A probabilistic complex mapping is of the form $p C M=\left\{\left(\mathrm{cm}_{i}, \operatorname{Pr}\left(\mathrm{cm}_{i}\right)\right) \mid\right.$ $i \in[1, n]\}$, where $\sum_{i=1}^{n} \operatorname{Pr}\left(c m_{i}\right)=1$.

Theorem 8.1. Let $\overline{p C M}$ be a schema probabilistic complex mapping between schemas $\bar{S}$ and $\bar{T}$. Let $D_{S}$ be an instance of $\bar{S}$. Let $Q$ be an SPJ query over $\bar{T}$. The data complexity and mapping complexity of computing $Q^{\text {table }}\left(D_{S}\right)$ with respect to $\overline{p C M}$ are PTIME. The data complexity of computing $Q^{\text {tuple }}\left(D_{S}\right)$ with respect to $\overline{p C M}$ is \#P-complete. The mapping complexity of computing $Q^{\text {tuple }}\left(D_{S}\right)$ with respect to $\overline{p C M}$ is in PTIME.

Conditional mappings: In practice, our uncertainty is often conditioned. For example, we may want to state that daytime-phone maps to work-phone with probability $60 \%$ if age $\leq 65$, and maps to home-phone with probability $90 \%$ if age $>65$.

We define a conditional $p$-mapping as a set $c p M=\left\{\left(p M_{1}, C_{1}\right), \ldots,\left(p M_{n}, C_{n}\right)\right\}$, where $p M_{1}, \ldots, p M_{n}$ are p-mappings, and $C_{1}, \ldots, C_{n}$ are pairwise disjoint conditions. Intuitively, for each $i \in[1, n], p M_{i}$ describes the probability distribution of possible mappings when condition $C_{i}$ holds. Conditional mappings make more sense for by-tuple semantics. The following theorem shows that our results carry over to such mappings.

Theorem 8.2. Let $\overline{c p M}$ be a schema conditional p-mapping between $\bar{S}$ and $\bar{T}$. Let $D_{S}$ be an instance of $\bar{S}$. Let $Q$ be an SPJ query over $\bar{T}$. The problem of computing $Q^{\text {tuple }}\left(D_{S}\right)$ with respect to $\overline{c p M}$ is in PTIME in the size of the mapping and \#P-complete in the size of the data.

\section{Related Work}

We are not aware of any previous work studying the semantics and properties of probabilistic schema mappings. Gal [11] used the top-K schema mappings obtained by a semi-automatic mapper to improve the precision of the top mapping, but did not address any of the issues we consider. Florescu et al. [10] were the first to advocate the use of probabilities in data integration. Their work used probabilities to model (1) a mediated schema with overlapping classes (e.g., DatabasePapers and AIPapers), (2) source descriptions stating the probability of a tuple being present in a source, and (3) overlap between data sources. While these are important aspects of many domains and should be incorporated into a data integration system, our focus here is different. De Rougement and Vieilleribiere [6] considered approximate data exchange in that they relaxed the constraints on the target schema, which is a different approach from ours.

There has been a flurry of activity around probabilistic and uncertain databases lately [4, 24, $5,3]$. Our intention is that a data integration system will be based on a probabilistic data model, and we leverage concepts from that work as much as possible. We also believe that uncertainty and lineage are closely related, in the spirit of [4], and that relationship will play a key role in data integration. We leave exploring this topic to future work.

\section{Discussion}

We now discuss several extensions to our study of probabilistic schema mapping. 
Top- $k$ query answering: Answering queries in the presence of probabilistic schema mappings in by-tuple semantics is \#-P complete, and so is quite expensive. In practice, users often want to see only the answers with the top- $k$ probabilities and are satisfied even if the probabilities of these tuples are not returned. Rather than first computing all answers and then returning the top- $k$ answer tuples, we can improve the efficiency by performing only the necessary query reformulations and executions at every step and halt when the top- $k$ answers are found.

Generating probabilities: To employ probabilistic mappings in resolving heterogeneity at the schema level, we must have a good method of generating probabilities for the mappings. This is possible as techniques for semi-automatic schema mapping are often based on Machine Learning techniques that at their core compute the confidence of correspondences they generate. However, such confidence is meant more as a ranking mechanism than true probabilities between candidates and is associated with attribute correspondences rather than candidate mappings. We plan to study how to generate from them probabilities for candidate mappings by pursuing maximum entropy.

Reasoning uncertainties: Another direction we would like to explore is to reason about the uncertainty on schema mappings between data sources and its effect on query answering. By analyzing the probabilities of the candidate mappings, we would like to find the critical parts (i.e., attribute correspondences) where it is most beneficial to expand more resources (human or otherwise) to improve schema mapping.

Probabilistic data integration: One of our future goals is to build a data integration system that supports uncertainty about mappings, data extracted from sources, and the exact meaning of keyword queries. Studying the theoretical underpinning of probabilistic mappings is the first step towards building such a system. In addition, we need to extend the current work in the community on probabilistic databases [24] to study how to efficiently answer queries in the presence of uncertainties in schemas and in data, and study how to translate a keyword query into structured queries by exploiting evidence obtained from the existing data and users' search and querying patterns.

\section{Summary}

In this paper we introduced probabilistic schema mappings, with which we are able to answer queries on heterogeneous data sources even if we have only a set of candidate mappings that may not be precise. We presented query answering algorithms for by-table and by-tuple semantics and studied the complexity of query answering. We also considered concise encoding of probabilistic mappings, with which we are able to improve the efficiency of query answering. Finally, we extended our definition to more powerful schema mapping languages and showed the extensibility of our approach.

\section{References}

[1] S. Abiteboul and O. Duschka. Complexity of answering queries using materialized views. In PODS, 1998.

[2] S. Agrawal, S. Chaudhuri, and G. Das. DBXplorer: A system for keyword-based search over relational databases. In $I C D E, 2002$. 
[3] L. Antova, C. Koch, and D. Olteanu. World-set decompositions: Expressiveness and efficient algorithms. In ICDT, 2007.

[4] O. Benjelloun, A. D. Sarma, A. Y. Halevy, and J. Widom. ULDBs: Databases with uncertainty and lineage. In $V L D B, 2006$.

[5] R. Cheng, S. Prabhakar, and D. V. Kalashnikov. Querying imprecise data in moving object environments. In ICDE, 2003.

[6] M. de Rougemont and A. Vieilleribiere. Approximate data exchange. In ICDT, 2007.

[7] X. Dong and A. Halevy. A platform for personal information management and integration. In CIDR, 2005.

[8] R. Fagin, P. G. Kolaitis, and L. Popa. Data exchange: Getting to the core. ACM Transactions on Database Systems, 30(1):174-201, 2005.

[9] R. Fagin, A. Lotem, and M. Naor. Optimal aggregation algorithms for middleware. In PODS, 2001.

[10] D. Florescu, D. Koller, and A. Levy. Using probabilistic information in data integration. In Proc. of $V L D B, 1997$.

[11] A. Gal. Managing uncertainty in schema matching with Top-K schema mappings. Journal on Data Semantics, 6, 2006.

[12] A. Y. Halevy. Answering queries using views: A survey. VLDB Journal, 10(4), 2001.

[13] A. Y. Halevy, N. Ashish, D. Bitton, M. J. Carey, D. Draper, J. Pollock, A. Rosenthal, and V. Sikka. Enterprise information integration: successes, challenges and controversies. In SIGMOD, 2005.

[14] A. Y. Halevy, M. J. Franklin, and D. Maier. Principles of dataspace systems. In PODS, 2006.

[15] A. Y. Halevy, A. Rajaraman, and J. J. Ordille. Data integration: The teenage years. In VLDB, 2006.

[16] V. Hristidis and Y. Papakonstantinou. DISCOVER: Keyword search in relational databases. In $V L D B, 2002$.

[17] M. Lenzerini. Data integration: A theoretical perspective. In Proc. of PODS, 2002.

[18] A. Y. Levy. Special issue on adaptive query processing. IEEE Data Eng. Bull., 23(2), 2000.

[19] C. Li, K. C.-C. Chang, and I. F. LLyas. Supporting ad-hoc ranking aggregates. In SIGMOD, 2006.

[20] J. Madhavan, S. Cohen, X. Dong, A. Halevy, S. Jeffery, D. Ko, and C. Yu. Navigating the seas of structured web data. In CIDR, 2007.

[21] J. Pearl. Probabilistic Reasoning in Intelligent Systems: Networks of Plausible Inference. Morgan Kaufmann Publishers, Inc., California, 1988.

[22] E. Rahm and P. A. Bernstein. A survey of approaches to automatic schema matching. VLDB Journal, 10(4):334-350, 2001. 
[23] C. Re, D. Suciu, and N. N. Dalvi. Query evaluation on probabilistic databases. IEEE Data Eng. Bull., 29(1), 2006.

[24] D. Suciu and N. N. Dalvi. Foundations of probabilistic answers to queries. In SIGMOD, 2005.

\section{Appendix A. Proof for Theorem 4.4}

Theorem 4.4. Let $Q$ be an SPJ query and let $\overline{p M}$ be a schema p-mapping.

The problem of finding the probability for a by-tuple answer to $Q$ with respect to $\overline{p M}$ is \#Pcomplete with respect to data complexity and is in PTIME with respect to mapping complexity.

Proof. We prove the theorem by proving three lemmas, stating that (1) the problem is in PTIME in the size of the mapping; (2) the problem is in \#P in the size of the data; (3) the problem is \#P-hard in the size of the data.

Lemma 12.1. Let $Q$ be an SPJ query and let $\overline{p M}$ be a schema p-mapping.

The problem of finding the probability for a by-tuple answer to $Q$ with respect to $\overline{p M}$ is in PTIME in the size of the mapping.

Proof. We can generate all answers in three steps. Let $T_{1}, \ldots, T_{l}$ be the relations mentioned in $Q$ 's FROM clause. Let $p M_{i}$ be the p-mapping associated with table $T_{i}$. Let $d_{i}$ be the number of tuples in the source table of $p M_{i}$.

1. For each $s e q^{1} \in \mathbf{s e q}_{d 1}\left(p M_{1}\right), \ldots, s e q^{l} \in \mathbf{s e q}_{d l}\left(p M_{l}\right)$, generate a target instance that is consistent with the source instance and $\overline{p M}$ as follows. For each $i \in[1, l]$, the target relation $T_{i}$ contains $d_{i}$ tuples, where the $j$-th tuple (1) is consistent with the $j$-th source tuple and the $j$-th mapping $m^{j}$ in $s e q^{i}$, and (2) contains null as the value of each attribute that is not involved in $m^{j}$.

2. For each target instance, answer $Q$ on the instance. Consider only the answer tuples that do not contain the null value and assign probability $\Pi_{i=1}^{l} \operatorname{Pr}\left(s e q^{i}\right)$ to the tuple.

3. For each distinct answer tuple, sum up its probabilities.

According to the definition of by-tuple answers, the algorithm generates all by-tuple answers. We now prove it takes polynomial time in the size of the mapping. Assume each p-mapping $p M_{i}$ contains $l_{i}$ mappings. Then, the number of instances generated in step 1 is $\Pi_{i=1}^{l} l_{i}^{d_{i}}$, polynomial in the size of $\overline{p M}$. In addition, the size of each generated target instance is linear in the size of the source instance. So the algorithm takes polynomial time in the size of the mapping.

Lemma 12.2. Let $Q$ be an SPJ query and let $\overline{p M}$ be a schema p-mapping.

The problem of finding the probability for a by-tuple answer to $Q$ with respect to $\overline{p M}$ is in \#P in the size of the data.

Proof. We prove the claim by reducing the problem to answering queries on disjunctive probabilistic databases, which is proved to be in \#P [23]. Before we describe the reduction, we first introduce probabilistic databases. 
Definition 12.3 (Probabilistic Database). A probabilistic database (p-database) $p D$ over a schema $\bar{R}$ is a set $\left\{\left(D_{1}, \operatorname{Pr}\left(D_{1}\right)\right), \ldots,\left(D_{n}, \operatorname{Pr}\left(D_{n}\right)\right)\right\}$, such that

- for $i \in[1, n], D_{i}$ is an instance of $\bar{R}$, and for $i \neq j, D_{i} \neq D_{j}$;

- $\operatorname{Pr}\left(D_{i}\right) \in[0,1]$ and $\sum_{i=1}^{n} \operatorname{Pr}\left(D_{i}\right)=1$.

Answers to queries over p-databases have probabilities associated with them. Specifically, let $Q$ be a query over $p D$, and let $t$ be a tuple. We denote by $\bar{D}(t)$ the subset of $p D$ such that for each $D \in \bar{D}(t), t \in Q(D)$. Let $p=\sum_{D \in \bar{D}(t)} \operatorname{Pr}(D)$. If $p>0$, we call $(t, p)$ a possible tuple in the answer of $Q$ on $p D$.

Given a SPJ query $Q$ and a p-database $p D$, we denote by $Q(p D)$ the set of all possible tuples in the answer of $Q$ on $p D$. Computing $Q(p D)$ takes polynomial time in the size of $p D$.

We next define a compact representation of p-databases, called disjunctive p-database, over which query answering is \# $\mathrm{P}$-complete in the size of the representation.

Definition 12.4 (Disjunctive P-Database). Let $R$ be a relation schema where there exists a set of attributes that together form the key of the relation. Let $p D_{R}^{\vee}$ be a set of tuples of $R$, each has a probability.

We say that $p D_{R}^{\vee}$ is a disjunctive p-database if for each key value that occurs in $p D_{R}^{\vee}$, the probabilities of the tuples with this key value sum up to 1.

In a disjunctive p-database, we consider tuples with the same key value as disjoint. Formally, let $k e y_{1}, \ldots, k e y_{n}$ be the set of all distinct key values in $p D_{R}^{\vee}$. For each $i \in[1, n]$, we denote by $d_{i}$ the number of tuples whose key value is $k e y_{i}$. Then, $p D_{R}^{\vee}$ defines a set of $\Pi_{i=1}^{n} d_{i}$ possible databases. Each possible database $(D, \operatorname{Pr}(D))$ contains $n$ tuples $t_{1}, \ldots, t_{n}$, such that (1) for each $i \in[1, n]$, the key value of $t_{i}$ is $k e y_{i}$; and $(2) \operatorname{Pr}(D)=\Pi_{i=1}^{n} \operatorname{Pr}\left(t_{i}\right)$.

We now describe the reduction. We reduce the problem of query answering with respect to probabilistic mappings to the problem of query answering on disjunctive p-databases. The reduction proceeds as follows.

For each relation $T$ that occurs in $Q$ and is involved in a p-mapping $p M=(S, T, \mathbf{m})$, generate the target instance as follows. The target instance is a disjunctive p-database with attributes in $T$ and a key column that is the key of the relation. For the $i$-th tuple $t_{s}$ in $S$ and each $m \in \mathbf{m}$, generate a target tuple $t_{t}$, such that (1) for each attribute correspondence $\left(a_{s}, a_{t}\right) \in m$, the value of $a_{t}$ is the same as the value of $a_{s}$ in $t_{s}$; (2) for each attribute $a_{t}$ in $T$ that is not involved in any attribute correspondence in $m$, the value of $a_{t}$ is null; and (3) the value of the key attribute is $i$. The probability of the tuple is $\operatorname{Pr}(m)$. Let $n$ be the number of tuples in $T$ and $l$ be the number of mappings in $p M$. Generating the target instance takes time $O(l \cdot n)$, polynomial in the size of the data and the mapping.

Let $D_{S}$ be a source instance and $p D_{T}$ be the generated target instance. We now prove $Q^{\text {tuple }}\left(D_{S}\right)=Q\left(p D_{T}^{\vee}\right)$, where we assume $Q\left(p D_{T}\right)$ does not return answers containing null values. We prove by showing that for each possible database $D_{T}$ of $p D_{T}^{\vee}$, there exists a mapping sequence seq, such that $\operatorname{Pr}\left(D_{T}\right)=\operatorname{Pr}(\mathrm{seq})$ and the set of tuples in $Q\left(D_{T}\right)$ is the same as the set of certain answers with respect to $s e q$, and vice versa.

I. Suppose $D_{T}$ contains tuples $t_{1}, \ldots, t_{n}$, where $t_{i}, i \in[1, n]$, has $i$ as the value of key. Then, $t_{i}$ is consistent with the $i$-th source tuple in $S$ and some mapping in $\mathbf{m}$. Let $m^{i}$ be this mapping. We then have a mapping sequence $<m^{1}, \ldots, m^{n}>$. Here, $\operatorname{Pr}\left(D_{T}\right)=\prod_{i=1}^{n} \operatorname{Pr}\left(t_{i}\right)=\prod_{i=1}^{n} \operatorname{Pr}\left(m^{i}\right)=\operatorname{Pr}(\operatorname{seq})$. 
Because $D_{T}$ is consistent with $D_{S}$ and seq, the certain answer $a$ must also be an answer tuple in $Q\left(D_{T}\right)$. We now prove for each tuple $a \in Q\left(D_{T}\right)$ and database $D_{T}^{\prime}$ that is consistent with $D_{S}$ and seq, $a \in Q\left(D_{T}^{\prime}\right)$ (so $a$ is a certain answer with respect to seq). Suppose the $i$-th tuple $t_{i} \in D_{T}$ is involved in generating $a$. Because $Q\left(p D_{T}^{\vee}\right)$ does not return null values, $t_{i}$ 's attributes that are not involved in $m^{i}$ do not contribute to generating $a$. Tuple $t_{i}^{\prime}$ has the same value with $t_{i}$ on all attributes that are involved in $m^{i}$. Thus, we can also generate $a$ with $t_{i}^{\prime}$ and $a \in Q\left(D_{T}^{\prime}\right)$.

II. Consider a mapping sequence $<m^{1}, \ldots, m^{n}>$. Consider the possible database $D_{T}$ where the $i$-th tuple has $i$ as the value of key and is consistent with $m^{i}$ and the $i$-th source tuple. Obviously, $\operatorname{Pr}($ seq $)=\operatorname{Pr}\left(D_{T}\right)$. We can prove tuples in $Q\left(D_{T}\right)$ are certain answers with respect to seq in the same way as in I.

Lemma 12.5. Consider the following query

Q: SELECT 'true'

FROM T, J, T'

WHERE T.a $=\mathrm{J} \cdot \mathrm{a}$ AND J.b $=\mathrm{T}^{\prime} \cdot \mathrm{b}$

Answering $Q$ with respect to $\overline{p M}$ is \#P-hard in the size of the data.

Proof. We prove the lemma by reducing the bipartite monotone 2-DNF problem to the above problem.

Consider a bipartite monotone 2-DNF problem where variables can be partitioned into $X=$ $\left\{x_{1}, \ldots, x_{m}\right\}$ and $Y=\left\{y_{1}, \ldots, y_{n}\right\}$, and $\varphi=C_{1} \vee \cdots \vee C_{l}$, where each clause $C_{i}$ has the form $x_{j} \wedge y_{k}, x_{j} \in X, y_{k} \in Y$. We construct the following query-answering problem.

$P$-mapping: Let $\overline{p M}$ be a schema p-mapping containing $p M$ and $p M^{\prime}$. Let $p M=(S, T, \mathbf{m})$ be a p-mapping where $S=<a>, T=<a^{\prime}>$ and

$$
\mathbf{m}=\left\{\left(\left\{\left(a, a^{\prime}\right)\right\}, .5\right),(\emptyset, .5)\right\}
$$

Let $p M^{\prime}=\left(S^{\prime}, T^{\prime}, \mathbf{m}^{\prime}\right)$ be a p-mapping where $S^{\prime}=<b>, T^{\prime}=<b^{\prime}>$ and

$$
\mathbf{m}^{\prime}=\left\{\left(\left\{\left(b, b^{\prime}\right)\right\}, .5\right),(\emptyset, .5)\right\} .
$$

Source data: The source relation $S$ contains $m$ tuples: $x_{1}, \ldots, x_{m}$. The source relation $S^{\prime}$ contains $n$ tuples: $y_{1}, \ldots, y_{n}$. The relation $J$ contains $l$ tuples. For each clause $C_{i}=x_{j} \wedge y_{k}$, there is a tuple $\left(x_{j}, y_{k}\right)$ in $J$.

Obviously the construction takes polynomial time. We now prove the answer to the query is tuple true with probability $\frac{\# \varphi}{2^{m+n}}$, where $\# \varphi$ is the number of variable assignments that satisfy $\varphi$. We prove by showing that for each variable assignment $v_{x 1}, \ldots, v_{x m}, v_{y 1}, \ldots, v_{y n}$ that satisfies $\varphi$, there exists a mapping sequence seq such that true is a certain answer with respect to $s e q$ and the source instance, and vice versa.

For each variable assignment $v_{x 1}, \ldots, v_{x m}, v_{y 1}, \ldots, v_{y n}$ that satisfies $\varphi$, there must exist $j$ and $k$ such that $v_{x j}=$ true, $v_{y k}=$ true, and there exists $C_{i}=x_{j} \wedge y_{k}$ in $\varphi$. We construct the mapping sequence for $p M$ such that for each $j \in[1, m]$, if $v_{x j}=$ true, $m^{j}=\left(\left\{\left(a, a^{\prime}\right)\right\}, .5\right)$, and if $v_{x k}=$ false, $m^{j}=(\emptyset, .5)$. We construct the mapping sequence for $p M^{\prime}$ such that for each $k \in[1, n]$, if $v_{y k}=$ true, $m^{\prime k}=\left(\left\{\left(b, b^{\prime}\right)\right\}, .5\right)$, and if $v_{y k}=$ false, $m^{\prime k}=(\emptyset, .5)$. Any target instance that is consistent with the source instance and $\left\{s e q, s e q^{\prime}\right\}$ contains $x_{j}$ in $T$ and $y_{k}$ in $T^{\prime}$. Since $C_{i} \in \varphi, J$ contains tuple $\left(x_{j}, y_{k}\right)$ and so true is a certain answer. 
For each mapping sequence $s e q$ for $p M$ and $s e q^{\prime}$ for $p M^{\prime}$, if true is a certain answer, there must exist $j \in[1, m]$ and $k \in[1, n]$, such that $x_{j}$ is in any target instance that is consistent with $S$ and $s e q, y_{k}$ is in any target instance that is consistent with $S^{\prime}$ and $s e q^{\prime}$, and there exists a tuple $\left(x_{j}, y_{k}\right)$ in $J$. Thus, $m^{j} \in \operatorname{seq}$ must be $\left(\left\{\left(a, a^{\prime}\right)\right\}, .5\right)$ and $m^{\prime k} \in s e q^{\prime}$ must be $\left(\left\{\left(b, b^{\prime}\right)\right\}, .5\right)$. We construct the assignments $v_{x 1}, \ldots, v_{x m}, v_{y 1}, \ldots, v_{y n}$ as follows. For each $j \in[1, m]$, if we have $m^{j}=\left(\left\{\left(a, a^{\prime}\right)\right\}, .5\right)$ in seq, $x_{j}=$ true; otherwise, $x_{j}=$ false. For each $k \in[1, n]$, if $m^{k}=\left(\left\{\left(b, b^{\prime}\right)\right\}, .5\right)$ in seq, $y_{k}=$ true; otherwise, $y_{k}=$ false. Obviously, the values of $x_{j}$ and $y_{k}$ are true, $\varphi$ contains a term $x_{j} \wedge y_{k}$, and so $\varphi$ is satisfied.

Counting the number of variable assignments that satisfy a bipartite monotone 2DNF boolean formula is \#P-complete. Thus, answering query $Q$ is \#P-hard.

Note that in Lemma $12.5 Q$ contains two joins. Indeed, as stated in the following conjecture, we suspect that even for a query that contains a single join, query answering is also \#P-complete. The proof is still an open problem.

Conjecture 12.6. Let $\overline{p M}$ be a schema p-mapping containing $p M$ and $p M^{\prime}$. Let $p M=(S, T, \mathbf{m})$ be a p-mapping where $S=<a, b>, T=<c>$ and

$$
\mathbf{m}=\{(\{(a, c)\}, .5),(\{(b, c)\}, .5)\} .
$$

Let $p M^{\prime}=\left(S^{\prime}, T^{\prime}, \mathbf{m}^{\prime}\right)$ be a p-mapping where $S^{\prime}=<d>, T^{\prime}=<e>$ and

$$
\mathbf{m}^{\prime}=\{(\{(d, e)\}, .5),(\emptyset, .5)\} .
$$

Consider the following query

Q: SELECT 'true'

FROM T1, T2

WHERE T1. $\mathrm{C}=\mathrm{T} 2 . \mathrm{e}$

Answering $Q$ with respect to $\overline{p M}$ is \#P-hard in the size of the data.

\section{Appendix B: Proofs for Other Results}

Theorem 4.1. Let $\overline{p M}$ be a schema p-mapping and let $Q$ be an SPJ query.

Answering $Q$ with respect to $\overline{p M}$ in by-table semantics is in PTIME in the size of the data and the mapping.

Proof. It is trivial that Algorithm BYTABLE computes all by-table answers. We now consider its time complexity by examining the time complexity of each step.

Step 1: Assume for each target relation $T_{i}, i \in[1, l]$, the involved p-mapping contains $n_{i}$ possible mappings. Then, the number of reformulated queries is $\prod_{i=1}^{l} n_{i}$, polynomial in the size of the mapping.

Given the restricted class of mappings we consider, we can reformulate the query as follows. For each of $T_{i}$ 's attributes $t$, if there exists an attribute correspondence $\left(S . s, T\right.$.t) in $m^{i}$, we replace $t$ everywhere with $s$; otherwise, the reformulated query returns empty result. Let $|Q|$ be the size of $Q$. Thus, reformulating a query takes time $O(|Q|)$, and the size of the reformulated query does not exceed the size of $Q$. 
Therefore, Step 1 takes time $O\left(\Pi_{i=1}^{l} n_{i} \cdot|Q|\right)$, which is polynomial in the size of the p-mapping and does not depend on the size of the data.

Step 2: Answering each reformulated query takes polynomial time in the size of the data and the number of answer tuples is polynomial in the size of the data. Because there are polynomial number of answer tuples and each occurs in the answers of no more than $\prod_{i=1}^{l} n_{i}$ queries, summing up the probabilities for each answer tuple takes time $O\left(\Pi_{i=1}^{l} n_{i}\right)$. Thus, Step 2 takes polynomial time in the size of the mapping and the data.

Theorem 4.2. Let $p G M$ be a general p-mapping between a source schema $\bar{S}$ and a target schema $\bar{T}$. Let $D_{S}$ be an instance of $\bar{S}$. Let $Q$ be an SPJ query with only equality conditions over $\bar{T}$.

The problem of computing $Q^{\text {table }}\left(D_{S}\right)$ with respect to $p G M$ is in PTIME in the size of the data and the mapping.

Proof. We proceed in two steps to return all by-table answers. In the first step, for each $g m_{i}, i \in$ $[1, n]$, we answer $Q$ according to $g m_{i}$ on $D_{S}$. The certain answer with regard to $g m_{i}$ has probability $\operatorname{Pr}\left(g m_{i}\right)$. SPJ queries with only equality conditions are conjunctive queries. According to [1], we can return all certain answers in polynomial time in the size of the data, and the number of certain answers is polynomial in the size of the data. Thus, the first step takes polynomial time in the size of the data and the mapping.

In the second step, we sum up the probabilities of each answer tuple. Because there are a polynomial number of answer tuples and each occurs in the answers of no more than $n$ reformulated queries, this step takes polynomial time in the size of the data and the mapping.

Lemma 4.6. Let $\overline{p M}$ be a schema p-mapping. Let $Q$ be an SPJ query and $Q_{m}$ be $Q$ 's mirror query with respect to $\overline{p M}$. Let $D_{S}$ be the source database and $D_{T}$ be the mirror target of $D_{S}$ with respect to $\overline{p M}$.

Then, $t \in Q^{\text {tuple }}\left(D_{S}\right)$ if and only if $t \in Q_{m}\left(D_{T}\right)$ and $t$ does not contain null value.

Proof. If: We prove $t \in Q^{\text {tuple }}\left(D_{S}\right)$ by showing that we can construct a mapping sequence seq such that for each target instance $D_{T}^{\prime}$ that is consistent with $D_{S}$ and seq, $t \in Q\left(D_{T}^{\prime}\right)$.

Assume query $Q$ (and so $Q_{m}$ ) contains $n$ subgoals (i.e., occurrences of tables in the From clause). Assume we obtain $t$ by joining $n$ tuples $t_{1}, \ldots, t_{n} \in D_{T}$, each in the relation of a subgoal. Consider a relation $R$ that occurs in $Q$. Assume $t_{k_{1}}, \ldots, t_{k_{l}},\left(k_{1}, \ldots, k_{l} \in[1, n]\right.$ ) are tuples of $R$ (for different subgoals). Let $p M \in \overline{p M}$ be the p-mapping where $R$ is the target and let $S$ be the source relation of $p M$. For each $j \in[1, l]$, we denote the id value of $t_{k_{j}}$ by $t_{k_{j}}$.id, and the mapping value of $t_{k_{j}}$ by $t_{k_{j}}$.mapping. Then, $t_{k_{j}}$ is consistent with the $t_{k_{j}}$.id-th source tuple in $S$ and the mapping $t_{k_{j}}$. mapping.

We construct the mapping sequence of $R$ for seq as follows: (1) for each $j \in[1, l]$, the mapping for the $t_{k_{j}}$.id-th tuple is $t_{k_{j}}$.mapping; (2) the rest of the mappings are arbitrary mappings in $p M$. To ensure the construction is valid, we need to prove that all tuples with the same id value have the same mapping value. Indeed, for every $j, h \in[1, l], j \neq h$, because $t_{k_{j}}$ and $t_{k_{h}}$ satisfy the predicate $\left(R_{1}\right.$.id $<>R_{2}$.id OR $R_{1}$.mapping $=R_{2}$.mapping $)$ in $Q_{m}$, if $t_{k_{j}}$. id $=t_{k_{h}}$. id then $t_{k_{j}}$.mapping $=t_{k_{h}}$. mapping.

We now prove for each target instance $D_{T}^{\prime}$ that is consistent with $D_{S}$ and seq, $t \in Q\left(D_{T}^{\prime}\right)$. For each $t_{i}, i \in[1, n]$, we denote by $t_{i}^{\prime}$ the tuple in $D_{T}^{\prime}$ that is consistent with the $t_{i}$. id-th source tuple and the $t_{i}$.mapping mapping. We denote by $R\left(t_{i}\right), i \in[1, n]$, the subgoal that $t_{i}$ belongs to. By the definition of mirror target and also because $t$ does not contain null value, for each attribute of $R\left(t_{i}\right)$ 
that is involved in $Q, t_{i}$ has non-null value, and so they are involved in the mapping $t_{i}$.mapping. Thus, $t_{i}^{\prime}$ has the same value for these attributes. So $t$ can be obtained by joining $t_{1}^{\prime}, \ldots, t_{n}^{\prime}$ and $t \in Q\left(D_{T}^{\prime}\right)$.

Only if: $t \in Q^{\text {tuple }}\left(D_{S}\right)$, so there exists a mapping sequence seq, such that for each $D_{T}^{\prime}$ that is consistent with $D_{S}$ and seq, $t \in Q\left(D_{T}^{\prime}\right)$. Consider such a $D_{T}^{\prime}$. Assume $t$ is obtained by joining tuples $t_{1}, \ldots, t_{n} \in D_{T}^{\prime}$, and for each $i \in[1, n], t_{i}$ is a tuple of subgoal $R_{i}$. Assume $t_{i}$ is consistent with source tuple $s_{i}$ and $m_{i}$. We denote by $t_{i}^{\prime}$ the instance in $D_{T}$ whose id value refers to $s_{i}$ and mapping value refers to $m_{i}$. Let $\bar{A}_{i}$ be the set of attributes of the subgoal $R_{i}$ that are involved in the query. Since $t$ is a "certain answer", all attributes in $\bar{A}_{i}$ must be involved in $m_{i}$. Thus, $t_{i}$ and $t_{i}^{\prime}$ have the same value for these attributes, and all predicates in $Q$ hold on $t_{1}^{\prime}, \ldots, t_{n}^{\prime}$.

Because $D_{T}^{\prime}$ is consistent with $D_{S}$, for every pair of tuples $t_{i}$ and $t_{j}, i, j \in[1, n]$, of the same relation, $t_{i}$ and $t_{j}$ are either consistent with different source tuples in $D_{S}$, or are consistent with the same source tuple and the same possible mapping. Thus, predicate $R_{1}$.id $<>R_{2}$.id OR $R_{1}$. mapping $=R_{2}$.mapping in the mirror query must hold on $t_{i}^{\prime}$ and $t_{j}^{\prime}$. Thus, $t \in Q_{m}\left(D_{T}\right)$.

Theorem 4.5: Given an SPJ query and a schema p-mapping, returning all by-tuple answers without probabilities is in PTIME with respect to data complexity.

Proof. According to the previous lemma, we can generate all by-tuple answers by answering the mirror query on the mirror target. The size of the mirror target is polynomial in the size of the data and the size of the p-mapping, so answering the mirror query on the mirror target takes polynomial time.

Lemma 4.8. Let $\overline{p M}$ be a schema p-mapping between $\bar{S}$ and $\bar{T}$. Let $Q$ be a non-p-join query over $\bar{T}$ and let $D_{S}$ be an instance of $\bar{S}$. Let $(t, \operatorname{Pr}(t))$ be a by-tuple answer with respect to $D_{S}$ and $\overline{p M}$. Let $\bar{T}(t)$ be the subset of $\mathbf{T}\left(D_{S}\right)$ such that for each $D \in \bar{T}(t), t \in Q^{\text {table }}(D)$. The following two conditions hold:

1. $\bar{T}(t) \neq \emptyset$;

2. $\operatorname{Pr}(t)=1-\Pi_{D \in \bar{T}(t),(t, p) \in Q^{\text {table }}(D)}(1-p)$.

Proof. We first prove (1). Let $T$ be the relation in $Q$ that is the target of a p-mapping and let $p M$ be the p-mapping. Let $s e q$ be the mapping sequence for $p M$ with respect to which $t$ is a by-tuple answer. Because $Q$ is a non-p-join query, there is no self join over $T$. So there must exist a target tuple, denoted by $t_{t}$, that is involved in generating $t$. Assume this target tuple is consistent with the $i$-th source tuple and a possible mapping $m \in p M$. We now consider the $i$-th tuple database $D_{i}$ in $\mathbf{T}\left(D_{S}\right)$. There is a target database that is consistent with $D_{i}$ and $m$, and the database also contains the tuple $t_{t}$. Thus, $t$ is a by-table answer with respect to $D_{i}$ and $m$, so $D_{i} \in \bar{T}(t)$ and $\bar{T}(t) \neq \emptyset$.

We next prove (2). We denote by $\bar{m}\left(D_{i}\right)$ the set of mappings in $\mathbf{m}$, such that for each $m \in \bar{m}\left(D_{i}\right)$, $t$ is a certain answer with respect to $D_{i}$ and $m$. For the by-table answer $\left(t, p_{i}\right)$ with respect to $D_{i}$, obviously $p_{i}=\sum_{m \in \bar{m}\left(D_{i}\right)} \operatorname{Pr}(m)$.

Let $d$ be the number of tuples in $D_{S}$. Now consider a sequence $s e q=<m^{1}, \ldots, m^{d}>$. As far as there exists $i \in[1, d]$, such that $m^{i} \in \bar{m}\left(D_{i}\right), t$ is a certain answer with respect to $D_{S}$ and seq. The probability of all sequences that satisfy the above condition is $1-\Pi_{i=1}^{d}\left(1-\sum_{m \in \bar{m}\left(D_{i}\right)} \operatorname{Pr}(m)\right)=$ $1-\Pi_{D \in \bar{T}(t),(t, p) \in Q^{\text {table }}(D)}(1-p)$. Thus, $\operatorname{Pr}(t)=1-\Pi_{D \in \bar{T}(t),(t, p) \in Q^{\text {table }}(D)}(1-p)$. 
Theorem 4.10. Let $\overline{p M}$ be a schema p-mapping and let $Q$ be a non-p-join query with respect to $\overline{p M}$.

Answering $Q$ with respect to $\overline{p M}$ in by-tuple semantics is in PTIME in the size of the data and the mapping.

Proof. We first prove Algorithm NonPJoin generates all by-tuple answers. According to Lemma 4.8, we should first answer $Q$ on each tuple database, and then compute the probabilities for each answer tuple. In Algorithm NonPJoin, since we introduce the id attribute and return its values, Step 2 indeed generates by-tuple answers for all tuple databases. Finally, Step 3 computes the probability according to (2) in the lemma.

We next prove Algorithm NonPJoin takes polynomial time in the size of the data and the size of the mapping. Step 1 goes through each possible mapping to add one more correspondence and thus takes linear time in the size of the mapping. In addition, the size of the revised mapping is linear in the size of the original mapping. Since Algorithm BYTABLE takes polynomial time in the size of the data and the mapping, so does Step 2 in Algorithm NonPJoIn; in addition, the size of the result is polynomial in the size of the data and the mapping. Step 3 of the algorithm goes over each result tuple generated from Step 2, doing the projection and computing the probabilities according to the formula, so takes linear time in the size of the result generated from Step 2, and so takes also polynomial time in the size of the data and the mapping.

Lemma 4.14. Let $\overline{p M}$ be a schema p-mapping. Let $Q$ be a projected p-join query with respect to $\overline{p M}$ and let $\bar{J}$ be a maximal p-join partitioning of $Q$. Let $Q_{J 1}, \ldots, Q_{J n}$ be the p-join components of $Q$ with respect to $\bar{J}$.

For any instance $D_{S}$ of the source schema of $\overline{p M}$ and result tuple $t \in Q^{\text {tuple }}\left(D_{S}\right)$, the following two conditions hold:

1. For each $i \in[1, n]$, there exists a single tuple $t_{i} \in Q_{J i}^{\text {tuple }}\left(D_{S}\right)$, such that $t_{1}, \ldots, t_{n}$ generate $t$ when joined together.

2. Let $t_{1}, \ldots, t_{n}$ be the above tuples. Then $\operatorname{Pr}(t)=\Pi_{i=1}^{n} \operatorname{Pr}\left(t_{i}\right)$.

Proof. We first prove (1). The existence of the tuple is obvious. We now prove there exists a single such tuple for each $i \in[1, n]$. A join component returns all attributes that occur in $Q$ and the join attributes that join partitions. The definition of maximal p-join partitioning guarantees for every two partitions, they are joined only on attributes that belong to relations involved in p-mappings. A projected-p-join query returns all such join attributes, so all attributes returned by the join component are also returned by $Q$. Thus, every two different tuples in the result of the join component lead to different query results.

We now prove (2). Since a partition in a join component contains at most one subgoal that is the target of a p-mapping in $\overline{p M}$, each p-join component is a non-p-join query. For each $i \in[1, n]$, let $\overline{s e q}_{i}$ be the mapping sequences with respect to which $t_{i}$ is a by-tuple answer. Obviously, $\operatorname{Pr}\left(t_{i}\right)=\sum_{s e q \in \overline{s e q}_{i}} \operatorname{Pr}(s e q)$.

Consider choosing a set of mapping sequences $\bar{S}=\left\{s e q_{1}, \ldots, s e q_{n}\right\}$, where $s e q_{i} \in \overline{s e q}_{i}$ for each $i \in[1, n]$. Obviously, $t$ is a certain answer with respect to $\bar{S}$. Because choosing different mapping sequences for different p-mappings are independent, the probability of $\bar{S}$ is $\Pi_{i=1}^{n} \operatorname{Pr}\left(s e q_{i}\right)$. Thus, 
we have

$$
\begin{aligned}
\operatorname{Pr}(t) & =\sum_{s e q_{1} \in \overline{s e q}_{1}, \ldots, s e q_{n} \in \overline{s e q}_{n}} \Pi_{i=1}^{n} \operatorname{Pr}\left(s e q_{i}\right) \\
& =\Pi_{i=1}^{n} \sum_{s e q_{i} \in \overline{s e q}_{i}} \operatorname{Pr}\left(s e q_{i}\right) \\
& =\Pi_{i=1}^{n} \operatorname{Pr}\left(t_{i}\right)
\end{aligned}
$$

This proves the claim.

Theorem 4.16. Let $\overline{p M}$ be a schema p-mapping and let $Q$ be a projected-p-join query with respect to $\overline{p M}$.

Answering $Q$ with respect to $\overline{p M}$ in by-tuple semantics is in PTIME in the size of the data and the mapping.

Proof. We first prove Algorithm PROJECTEDPJoIn generates all by-tuple answers for projected-pjoin queries. First, it is trivial to verify that the partitioning generated by step 1 satisfies the two conditions of a p-join partitioning and is maximal. Then, step 2 and step 3 compute the probability for each by-tuple answer according to Lemma 4.14 .

We next prove it takes polynomial time in the size of the mapping and in the size of the data. Step 1 takes time polynomial in the size of the query, and is independent of the size of the mapping and the data. The number of p-join components is linear in the size of the query and each is smaller than the original query. Since Algorithm NonPJoin takes polynomial time in the size of the data and the size of the mapping, Step 2 takes polynomial time in the size of the mapping and the size of the data too, and the size of each result is polynomial in size of the data and the mapping. Finally, joining the results from Step 2 takes polynomial time in the size of the results, and so also polynomial in the size of the data and the mapping.

Theorem 6.3. Let $\overline{g p M}$ be a schema group p-mapping and let $Q$ be an SPJ query. The mapping complexity of answering $Q$ with respect to $\overline{g p M}$ in both by-table semantics and by-tuple semantics is in PTIME.

Proof. We first consider by-table semantics and then consider by-tuple semantics. For each semantics, we prove the theorem by first describing the query-answering algorithm, then proving the algorithm generates the correct answer, and next analyzing the complexity of the algorithm.

By-table semantics: I. First, we describe the algorithm that we answer query $Q$ with respect to the group p-mapping $\overline{g p M}$. Assume $Q$ 's FROM clause contains relations $T_{1}, \ldots, T_{l}$. For each $i \in[1, l]$, assume $T_{i}$ is involved in group p-mapping $g p M_{i}$, which contains $g_{i}$ groups (if $T_{i}$ is not involved in any group p-mapping, we assume it is involved in an identity p-mapping that corresponds each attribute with itself). The algorithm proceeds in five steps.

Step 1. We first partition all target attributes for $T_{1}, \ldots, T_{l}$ as follows. First, initialize each partition to contain attributes in one group (there are $\sum_{i=1}^{l} g_{i}$ groups). Then, for each pair of attributes $a_{1}$ and $a_{2}$ that occur in the same predicate in $Q$, we merge the two groups that $t_{1}$ and $t_{2}$ belong to. We call the result partitioning an independence partitioning with respect to $Q$ and $\overline{g p M}$.

Step 2. For each partition $p$ in an independence partitioning, if $p$ contains attributes that occur in $Q$, we generate a sub-query of $Q$ as follows. (1) The SELECT clause contains all variables in $Q$ that are included in $p$, and an id column for each relation that is involved in $p$ (we assume each tuple 
contains an identifier column id; in practice, we can use the key attribute of the tuple in place of id); (2) The FROM clause contains all relations that are involved in $p$; and (3) The WHERE clause contains only predicates that involve attributes in $p$. The query is called the independence query of $p$ and is denoted by $Q(p)$.

Step 3. For each partition $p$, let $p M_{1}, \ldots, p M_{n}$ be the p-mappings for the group of attributes involved in $p$. For each $m^{1} \in p M_{1}, \ldots, m^{n} \in p M_{n}$, rewrite $Q(p)$ regarding $m^{1}, \ldots, m^{n}$ and answer the rewritten query on the source data. For each returned tuple, assign $\Pi_{i=1}^{n} m^{i}$ as the probability and add $n$ columns mapping ${ }_{1}, \ldots$, mapping ${ }_{n}$, where the column mapping $i, i \in[1, n]$, has the identifier for $m_{i}$ as the value. Union all result tuples.

Step 4. Join the results of the sub-queries on the id attributes. Assume the result tuple $t$ is obtained by joining $t_{1}, \ldots, t_{k}$, then $\operatorname{Pr}(t)=\prod_{i=1}^{k} \operatorname{Pr}\left(t_{k}\right)$.

Step 5. For tuples that have the same values, assuming to be tuple $t$, for attributes on $Q$ 's returned attributes but different values for the mapping attributes, sum up their probabilities as the probability for the result tuple $t$.

II. We now prove the algorithm returns the correct by-table answers. For each result answer tuple $a$, we should add up the probabilities of the possible mappings with respect to which $a$ is generated. This is done in Step 5. So we only need to show that given a specific combination of mappings, the first four steps generate the same answer tuples as with normal p-mappings. The partitioning in Step 1 guarantees that different independence queries involve different p-mappings and so Step 2 and 3 generate the correct answer for each independence query. Step 4 joins results of the sub-queries on the id attributes; thus, for each source tuple, the first four steps generate the same answer tuple as with normal p-mappings. This proves the claim.

III. We next analyze the time complexity of the algorithm. The first two steps take polynomial time in the size of the mapping and the number of sub-queries generated by Step 2 is polynomial in the size of the mapping. Step 3 answers each sub-query in polynomial time in the size of the mapping and the result is polynomial in the size of the mapping. Step 4 joins a set of results from Step 3, where the number of the results and the size of each result is polynomial in the size of the mapping, so it takes polynomial time in the size of the mapping too and the size of the generated result is also polynomial in the size of the mapping. Finally, Step 5 takes polynomial time in the size of the result generated in Step 4 and so takes polynomial time in the size of the mapping. This proves the claim.

By-tuple semantics: First, we describe the algorithm that we answer query $Q$ with respect to the group p-mapping $\overline{g p M}$. The algorithm proceeds in five steps and the first two steps are the same as in by-table semantics.

Step 3. For each partition $p$, let $p M_{1}, \ldots, p M_{n}$ be the p-mappings for the group of attributes involved in $p$. For each mapping sequence seq over $p M_{1}, \ldots, p M_{n}$, answer $Q(p)$ with respect to $s e q$ in by-tuple semantics. For each returned tuple, assign $\operatorname{Pr}(s e q)$ as the probability and add a column seq with an identifier of $s e q$ as the value.

Step 4. Join the results of the sub-queries on the id attributes. Assume the result tuple $t$ is obtained by joining $t_{1}, \ldots, t_{k}$, then $\operatorname{Pr}(t)=\prod_{i=1}^{k} \operatorname{Pr}\left(t_{k}\right)$.

Step 5. Let $t_{1}, \ldots, t_{n}$ be the tuples that have the same values, tuple $t$, for attributes on $Q$ 's returned attributes but different values for the seq attributes, sum up their probabilities as the probability for the result tuple $t$.

We can verify the correctness of the algorithm and analyze the time complexity in the same way as in by-table semantics. 
Proposition 6.4. For each $n \geq 1, \mathcal{M}_{S T}^{n+1} \subset \mathcal{M}_{S T}^{n}$.

Proof. We first prove for each $n \geq 1, \mathcal{M}_{S T}^{n+1} \subseteq \mathcal{M}_{S T}^{n}$, and then prove there exists an instance in $\mathcal{M}_{S T}^{n}$ that does not have an equivalent instance in $\mathcal{M}_{S T}^{n+1}$.

(1) We prove $\mathcal{M}_{S T}^{n+1} \subseteq \mathcal{M}_{S T}^{n}$ by showing for each $(n+1)$-group p-mapping we can find a $n$-group p-mapping equivalent to it. Consider an instance $g p M=(S, T, \overline{p M}) \in \mathcal{M}_{S T}^{n+1}$, where $\overline{p M}=\left\{p M_{1}, \ldots, p M_{n+1}\right\}$. We show how we can construct an instance $g p M^{\prime} \in \mathcal{M}_{S T}^{n}$ that is equivalent to gp $M$. Consider merging $p M_{1}=\left(S_{1}, T_{1}, \mathbf{m}_{1}\right)$ and $p M_{2}=\left(S_{2}, T_{2}, \mathbf{m}_{2}\right)$ and generating a probabilistic mapping $p M_{1-2}=\left(S_{1} \cup S_{2}, T_{1} \cup T_{2}, \mathbf{m}_{1-2}\right)$, where $\mathbf{m}_{1-2}$ includes the Cartesian product of the mappings in $\mathbf{m}_{1}$ and $\mathbf{m}_{2}$. Consider the $n$-group p-mapping $g p M^{\prime}=\left(S, T, \overline{p M^{\prime}}\right)$, where $\overline{p M^{\prime}}=\left\{p M_{1-2}, p M_{3}, \ldots, p M_{n+1}\right\}$. Then, $g p M$ and $g p M^{\prime}$ describe the same mapping.

(2) We now show how we can construct an instance in $\mathcal{M}_{S T}^{n}$ that does not have an equivalent instance in $\mathcal{M}_{S T}^{n+1}$. If $S$ and $T$ contain less than $n$ attributes, $\mathcal{M}_{S T}^{n}=\emptyset$ and the claim holds. Otherwise, we partition attributes in $S$ and $T$ into $\left\{\left\{s_{1}\right\}, \ldots,\left\{s_{n-1}\right\},\left\{s_{n}, \ldots, s_{m}\right\}\right\}$ and $\left\{\left\{t_{1}\right\}, \ldots,\left\{t_{n-1}\right\},\left\{t_{n}, \ldots, t_{l}\right\}\right\}$. Without losing generality, we assume $m \leq l$. For each $i \in[1, n-1]$, we define

$$
\mathbf{m}_{i}=\left\{\left(\left\{\left(s_{i}, t_{i}\right)\right\}, 0.8\right),(\emptyset, 0.2)\right\} .
$$

In addition, we define

$$
\mathbf{m}_{n}=\left\{\left(\left\{\left(s_{n}, t_{n}\right)\right\}, \frac{1}{(m-n+1)}\right), \ldots,\left(\left\{\left(s_{m}, t_{n}\right)\right\}, \frac{1}{(m-n+1)}\right)\right\} .
$$

We cannot further partition $S$ into $n+1$ subsets such that attributes in different subsets correspond to different attributes in $T$. Thus, we cannot find a $(n+1)$-group p-mapping equivalent to it.

Theorem 6.5. Given a p-mapping $p M=(S, T, \mathbf{m})$, we can find in polynomial time in the size of $p M$ the maximal $n$ and an $n$-group p-mapping $g p M$, such that $g p M$ is equivalent to $p M$.

Proof. We prove the theorem by first presenting an algorithm that finds the maximal $n$ and the equivalent $n$-group p-mapping $g p M$, then proving the correctness of the algorithm, and next analyzing its time complexity.

I. We first present the algorithm that takes a p-mapping $p M=(S, T, \mathbf{m})$, finds the maximal $n$ and the $n$-group p-mapping that is equivalent to $p M$.

Step 1. First, partition attributes in $S$ and $T$. Initialize the partitions such that each contains a single attribute in $S$ or $T$. Then for each attribute correspondence $(s, t)$ occurring in a possible mapping, if $s$ and $t$ are in different partitions, merge the two partitions. Let $\mathcal{P}=\left\{p_{1}, \ldots, p_{n}\right\}$ be the result partitioning.

Step 2. For each partition $p_{i}, i \in[1, n]$, and each $m \in \mathbf{m}$, select the correspondences in $m$ that involve only attributes in $p_{i}$, use them to construct a sub-mapping, and assign $\operatorname{Pr}(m)$ to the submapping. We compute the marginal probability of each sub-mapping.

Step 3. For each partition $p_{i}, i \in[1, n]$, examine if its possible mappings are independent of the possible mappings for the rest of the partitions. Specifically, for each partition $p_{j}, j>i$, if there exists a possible mapping $m$ for $p_{i}$ and a possible mapping $m^{\prime}$ for $p_{j}$, such that $\operatorname{Pr}\left(m \mid m^{\prime}\right) \neq \operatorname{Pr}(m)$, merge $p_{i}$ into $p_{j}$. For the new partition $p_{j}$, update its possible sub-mappings and their marginal probabilities. Step 3 generates a set of partitions, each with a set of sub-mappings and their probabilities.

Step 4. Each partition generated in Step 3 is associated with a p-mapping. The set of all p-mappings forms the group p-mapping $g p M$ that is equivalent to $p M$. 
II. We now prove the correctness of the algorithm. It is easy to prove $g p M$ is equivalent to $p M$. Assume $g p M$ is an $n$-group p-mapping. We next prove $n$ is maximal. Consider another group p-mapping $g p M^{\prime}$. We now prove for each p-mapping in $g p M^{\prime}$, it either contains all attributes in a partition generated in Step 3 or contains none of them. According to the definition of group p-mapping, each p-mapping in $g p M^{\prime}$ must contain either all attributes or none of the attributes in a partition in $\mathcal{P}$. In addition, every two partitions in $\mathcal{P}$ that are merged in Step 3 are not independent and have to be in the same p-mapping in $g p M^{\prime}$ too. This proves the claim.

III. We next consider the time complexity of the algorithm. Let $m$ be the number of mappings in $p M$, and $a$ be the minimum number of attributes in $R$ and in $S$. Step 1 considers each attribute correspondence in each possible mapping. A mapping contains no more than $a$ attribute correspondences, so Step 1 takes time $O(m a)$. Step 2 considers each possible mapping for each partition to generate sub-mappings. The number of partitions cannot exceed $a$, so Step 2 also takes time $O(m a)$. Step 3 considers each pair of partitions. and takes time $O\left(m a^{2}\right)$. Finally, Step 4 outputs the results and takes time $O(m a)$. Overall, the algorithm takes time $O\left(m a^{2}\right)$, which is polynomial in the size of the full-distribution instance.

Theorem 6.9. Let $\overline{p C}$ be a schema p-correspondence, and $Q$ be an SPJ query. Then, $Q$ is pmapping independent with respect to $\overline{p C}$ if and only if for each $p C \subseteq \overline{p C}, Q$ is a single-attribute query with respect to $p C$.

Proof. We prove for the case when there is a single p-correspondence in $\overline{p C}$ and it is easy to generalize our proof to the case when there are multiple p-correspondences in $\overline{p C}$.

If: Let $p M_{1}$ and $p M_{2}$ be two p-mappings over $S$ and $T$ where $p C\left(p M_{1}\right)=p C\left(p M_{2}\right)$. Let $D_{S}$ be a database of schema $S$. Consider a query $Q$ over $T$. Let $t_{j}$ be the only attribute involved in query $Q$. We prove $Q\left(D_{S}\right)$ is the same with respect to $p M_{1}$ and $p M_{2}$ in both by-table and by-tuple semantics.

We first consider by-table semantics. Assume $S$ has $n$ attributes $s_{1}, \ldots, s_{n}$. We partition all possible mappings in $p M_{1}$ into $\bar{m}_{0}, \ldots, \bar{m}_{n}$, such that for any $m \in \bar{m}_{i}, i \in[1, n], m$ maps attribute $s_{i}$ to $t_{j}$, and for any $m \in \bar{m}_{0}, m$ does not map any attribute in $S$ to $t_{j}$. Thus, for each $i \in[1, n], \operatorname{Pr}\left(\bar{m}_{i}\right)=\operatorname{Pr}\left(c_{i j}\right)$.

Consider a tuple $t$. Assume $t$ is an answer tuple with respect to a subset of possible mappings $\bar{m} \subseteq \mathbf{m}$. Because $Q$ contains only attribute $t_{j}$, for each $i \in[0, n]$, either $\bar{m}_{i} \subseteq \bar{m}$ or $\bar{m}_{i} \cap \bar{m}_{=} \emptyset$. Let $\bar{m}_{k_{1}}, \ldots, \bar{m}_{k_{l}}, k_{1}, \ldots, k_{l} \in[0, n]$, be the subsets of $\bar{m}$ such that $\bar{m}_{k_{j}} \subseteq \bar{m}$ for any $j \in[1, l]$. We have

$$
\operatorname{Pr}(t)=\sum_{i=1}^{l} \operatorname{Pr}\left(\bar{m}_{k_{i}}\right)=\sum_{i=1}^{l} \operatorname{Pr}\left(c_{k_{i} j}\right) .
$$

Now consider $p M_{2}$. We partition its possible mappings in the same way and obtain $\bar{m}_{0}^{\prime}, \ldots, \bar{m}_{n}^{\prime}$. Since $Q$ contains only attribute $t_{j}$, for each $i \in[0, n]$, the result of $Q$ with respect to $m^{\prime} \in \bar{m}_{i}^{\prime}$ is the same as the result with respect to $m \in \bar{m}_{i}$. Therefore, the probability of $t$ with respect to $p M_{2}$ is

$$
\operatorname{Pr}(t)^{\prime}=\sum_{i=1}^{l} \operatorname{Pr}\left(\bar{m}_{k_{i}}^{\prime}\right)=\sum_{i=1}^{l} \operatorname{Pr}\left(c_{k_{i} j}\right) .
$$

Thus, $\operatorname{Pr}(t)=\operatorname{Pr}(t)^{\prime}$ and this proves the claim.

We can prove the claim for by-tuple semantics in a similar way where we partition mapping sequences. We omit the proof here. 
Only if: We prove by showing that for every query $Q$ that contains more than one attribute in a relation being involved in a p-correspondence, there exist p-mappings $p M_{1}$ and $p M_{2}$ and source instance $D_{S}$, such that $Q\left(D_{S}\right)$ obtains different results with respect to $p M_{1}$ and $p M_{2}$.

Assume query $Q$ contains attributes $a^{\prime}$ and $b^{\prime}$ of $T$. Consider two p-mappings $p M_{1}$ and $p M_{2}$, where

$$
\begin{aligned}
& p M_{1}=\left\{\left(\left\{\left(a, a^{\prime}\right),\left(b, b^{\prime}\right)\right\}, .5\right),\left(\left\{\left(a, a^{\prime}\right)\right\}, .3\right),\left(\left\{\left(b, b^{\prime}\right)\right\}, .2\right)\right\} \\
& p M_{2}=\left\{\left(\left\{\left(a, a^{\prime}\right),\left(b, b^{\prime}\right)\right\}, .6\right),\left(\left\{\left(a, a^{\prime}\right)\right\}, .2\right),\left(\left\{\left(b, b^{\prime}\right)\right\}, .1\right),(\emptyset, .1)\right\}
\end{aligned}
$$

One can verify that $p C\left(p M_{1}\right)=p C\left(p M_{2}\right)$.

Consider a database $D_{S}$, such that for each tuple of the source relation in $p M_{1}$ and $p M_{2}$, the values for attributes $a$ and $b$ satisfy the predicates in $Q$. Since only when the possible mapping $\left\{\left(a, a^{\prime}\right),\left(b, b^{\prime}\right)\right\}$ is applied can we generate valid answer tuples, but the possible mapping $\left\{\left(a, a^{\prime}\right),\left(b, b^{\prime}\right)\right\}$ has different probabilities in $p M_{1}$ and $p M_{2}, Q\left(D_{S}\right)$ obtains different results with respect to $p M_{1}$ and $p M_{2}$ in both semantics.

Corollary 6.11. Let $\overline{p C}$ be a schema p-correspondence, and $Q$ be a p-mapping independent SPJ query with respect to $\overline{p C}$. The mapping complexity of answering $Q$ with respect to $\overline{p C}$ in both by-table semantics and by-tuple semantics is in PTIME.

Proof. By-table: We revise algorithm BY-TABLE, which takes polynomial time in the size of the schema p-mapping, to compute answers with respect to schema p-correspondences. At the place where we consider a possible mapping in the algorithm, we revise to consider a possible attribute correspondence. Obviously the revised algorithm generates the correct by-table answers and takes polynomial time in the size of the mapping.

By-tuple: We revise the algorithm in the proof of Theorem 4.4, which takes polynomial time in the size of the schema p-mapping, to compute answers with respect to schema p-correspondences. Everywhere we consider a possible mapping in the algorithm, we revise to consider a possible attribute correspondence. Obviously the revised algorithm generates the correct by-tuple answers and takes polynomial time in the size of the mapping.

Theorem 6.13. There exists a schema p-mapping $\overline{p M}$ and a query $Q$, such that answering $Q$ with respect to $\overline{p M}$ in by-table semantics takes exponential time in size of $\overline{p M}$ 's Bayes-Net representation.

Proof. Consider $p M$ in Example 6.12. Consider the following query:

SELECT t $1, \ldots$, tn

FROM T

Consider a source instance $D_{S}$ with one tuple, where each attribute value in the tuple is distinct. There are $2^{n}$ tuples in $Q^{\text {table }}\left(D_{S}\right)$. To enumerate all these answers takes time $O\left(2^{n}\right)$, which is exponential in the size of $p M$ 's Bayes-Net representation.

Theorem 8.1. Let $\overline{p C M}$ be a schema probabilistic complex mapping between schemas $\bar{S}$ and $\bar{T}$. Let $D_{S}$ be an instance of $\bar{S}$. Let $Q$ be an SPJ query over $\bar{T}$. The data complexity and mapping complexity of computing $Q^{\text {table }}\left(D_{S}\right)$ with respect to $\overline{p C M}$ are PTIME. The data complexity of computing $Q^{\text {tuple }}\left(D_{S}\right)$ with respect to $\overline{p C M}$ is \#P-complete. The mapping complexity of computing $Q^{\text {tuple }}\left(D_{S}\right)$ with respect to $\overline{p C M}$ is in PTIME. 
Proof. We prove the theorem by showing that we can construct a normal schema p-mapping from $\overline{p C M}$ and answer a query with respect to the normal p-mapping. For each $p C M \in \overline{p C M}$ between source $S\left(s_{1}, \ldots, s_{m}\right)$ and target $T\left(t_{1}, \ldots, t_{n}\right)$, we construct a normal p-mapping $p M=\left(S^{\prime}, T^{\prime}, m\right)$ as follows. The source $S^{\prime}$ contains all elements of the power set of $\left\{s_{1}, \ldots, s_{m}\right\}$ and the target $T^{\prime}$ contains all elements of the power set of $\left\{t_{1}, \ldots, t_{n}\right\}$. For each complex mapping $\mathrm{cm} \in p C M$, we construct a mapping $m$ such that for each set correspondence between $S$ and $T$ in $\mathrm{cm}, m$ contains an attribute correspondence between the corresponding sets in $S^{\prime}$ and $T^{\prime}$. Because each attribute occurs in one correspondence in $\mathrm{cm}, \mathrm{m}$ is a one-to-one mapping. The result $p M$ contains the same number of possible mappings and each mapping contains the same number of correspondences as $p C M$. We denote the result schema p-mapping by $\overline{p M}$. Query answering with respect to $\overline{p C M}$ gets the same result as with respect to $\overline{p M}$ and so the complexity results for normal schema p-mappings carry over.

Theorem 8.2. Let $\overline{c p M}$ be a schema conditional p-mapping between $\bar{S}$ and $\bar{T}$. Let $D_{S}$ be an instance of $\bar{S}$. Let $Q$ be an SPJ query over $\bar{T}$. The problem of computing $Q^{\text {tuple }}\left(D_{S}\right)$ with respect to $\overline{c p M}$ is in PTIME in the size of the mapping and \#P-complete in the size of the data.

Proof. By-tuple query answering with respect to schema conditional p-mappings is essentially the same as that with respect to normal p-mappings, where for each source tuple, we first decide which condition it satisfies and then consider applying possible mappings associated with that condition. Thus, the complexity of by-tuple query-answering with respect to normal schema p-mappings carries over. 